OPEN ACCESS

Edited by:

Akio Adachi,

Kansai Medical University, Japan

Reviewed by:

Miguel Lopez-Ferber,

École des Mines d'Alès, France

Hayato Yamada,

Nagoya University, Japan

*Correspondence:

Qi Tang

tangqi1224@163.com

Specialty section:

This article was submitted to

Virology,

a section of the journal

Frontiers in Microbiology

Received: 29 July 2019 Accepted: 11 November 2019

Published: 26 November 2019

Citation:

Li G, Qi X, Chen H, Hu Z, Chen F, Deng L, Guo Z, Chen K and Tang $Q$ (2019) The Motif of ${ }^{76}$ KRKCSK in Bm65 Is an Efficient Nuclear Localization Signal Involved in Production of Infectious Virions.

Front. Microbiol. 10:2739.

doi: 10.3389/fmicb.2019.02739

\title{
The Motif of ${ }^{76} \mathrm{KRKCSK}$ in Bm65 Is an Efficient Nuclear Localization Signal Involved in Production of Infectious Virions
}

\section{Guohui Li, Xinyu Qi, Huiqing Chen, Zhaoyang Hu, Fangying Chen, Liang Deng, Zhongjian Guo, Keping Chen and Qi Tang*}

Institute of Life Sciences, Jiangsu University, Zhenjiang, China

orf65 (Bm65) of Bombyx mori nucleopolyhedrovirus (BmNPV) codes for a putative 104-amino-acid protein containing three cysteine residues with a putative molecular mass of $12.2 \mathrm{kDa}$. Previous studies have showed that Bm65 accumulates mainly in nucleus and involved in the repair of UV-damaged DNA. However, the mechanism of nuclear import of Bm65 remains unclear. In this study, a SDS-stable Bm65 tetramer was found in BmNPV-infected BmN cells, and alanine substitutions for the three cysteine residues did not affect the formation of Bm65 tetramer. Additionally, a basic amino acid cluster of the Bm65 protein was identified as an efficient nuclear localization signal (NLS). Firstly, transient expression of GFP-fused truncated Bm65 variants revealed that the ${ }^{76}$ KRKCSK motif functions as the NLS. This was also confirmed by alanine substitution in the ${ }^{76} \mathrm{KRKCSK}$ motif, which caused attenuated nuclear localization of Bm65. Next, the ${ }^{76}$ KRKCSK motif-mutated bacmid was generated and the ${ }^{76}$ KRKCSK motif was also found to be important for nuclear localization of Bm65 in BmNPV-infected conditions. Lastly, analyses of flag-tagged Bm65 expressing bacmids revealed that the mutations in ${ }^{76} \mathrm{KRKCSK}$ motif did not affect the synthesis of Bm65 tetramer, but severely impaired production levels of infectious virions. In conclusion, Bm65 exists in mainly a tetrameric form in virus-infected cells, which may be involved with production levels of infectious virions.

Keywords: BmNPV Bm65, Bm65 tetramer, point mutation, Bm65 truncation, nuclear localization signal

\section{INTRODUCTION}

Baculoviruses are within a group of enveloped, double-stranded DNA insect viruses with large, closed and circular genomes ranging in size from 80 to $180 \mathrm{kbp}$ (Herniou et al., 2003; van Oers and Vlak, 2007; Yin et al., 2015), which are characterized by a biphasic infection phase with production of two types of progeny virions during the viral life cycle. The two phenotypes are budded viruses (BVs) and occlusion-derived viruses (ODVs), which carry identical genetic information but differ structurally and functionally (Slack and Arif, 2007; Cheng and Lynn, 2009). BVs are produced at the initial stage of baculovirus life cycle and are responsible for the spread of infection from cell to cell, 
while ODVs are produced in the late stage of viral life cycle and mediate the horizontal transmission among insects (Clem and Passarelli, 2013; Rohrmann, 2013).

Bombyx mori nucleopolyhedrovirus (BmNPV) belongs to the Alphabaculovirus genus, Baculoviridae family and infects exclusively silkworms. BmNPV epizootics result in serious losses in silk production. Therefore, it is necessary to clarify the mechanism of BmNPV infection at the molecular level, which is helpful to control viral spread among silkworms. The functions of most viral genes in the process of BmNPV propagation, interactions between BmNPV and silkworm, and the innate response against BmNPV invasion have been extensively studied since Gomi et al. (1999) published the sequence of BmNPV genome (Ono et al., 2012; Qin et al., 2012; Xue et al., 2012). Additionally, the mechanism of BmNPV proliferation in silkworm has been gradually elucidated. Like other viruses, the propagation of BmNPV in host cells is inevitably involved with an important number of virus-encoded proteins that are required to generate progeny virions. Previous research reported that Bm65 is an early gene by transcriptional analysis (Tang et al., 2013), indicating that Bm65 may be involved with viral propagation. Tang et al. (2015, 2017) further reported that Bm65 localizes mainly in nucleus and is involved with the repair of UV-damaged DNA. However, the size of Bm65 in BmNPV-infected conditions remains unclear. So, we want to check the expression of Bm65 in BmNPVinfected $\mathrm{BmN}$ cells. Meanwhile, the mechanism of nuclear import and the impact of Bm65 on viral propagation are demonstrated in the study.

In the current study, a series of transient expression plasmids, including Bm65 truncations and point mutations in Bm65, were fused with enhanced green fluorescent protein (EGFP) respectively. The target DNA fragments were under control of ie1 promoter for expression of fusion protein tagged with EGFP. These plasmids were transfected into $\mathrm{BmN}$ cells to examine the intracellular distribution of fluorescent signal. Furthermore, the effect of mutations in Bm65 ${ }^{76} \mathrm{KRKCSK}$ motif on viral propagation was further evaluated by analysis of production of infectious virions.

\section{MATERIALS AND METHODS}

\section{Bacmid, Virus, Plasmids, Bacterial Strains, and Cells}

Bombyx mori nucleopolyhedrovirus (BmNPV) bacmid (Bmbacmid) with a deletion of $\mathrm{Bm} 65\left(\mathrm{Bm}^{\mathrm{Bm} 65 \mathrm{KO}}\right)$ was generated as previously described (Tang et al., 2013), and propagated in Escherichia coli strain DH10B harboring the pMON7124 helper plasmid. vBm ${ }^{\text {(PBm65-Bm65-egfp) }}$ was made by Tang et al. (2015) and used as a control of wild type in the study. Plasmid of pFastHTB- $\mathrm{P}_{\mathrm{Bm} 65}-\mathrm{Bm} 65-\mathrm{eg} p$ was constructed as previously described by Tang et al. (2015). Recombinant plasmid pFastHTB-

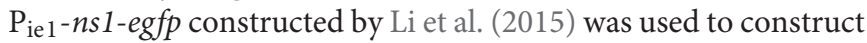
serial Bm65 truncations fused with egfp to study intracellular distribution of fluorescence signals in $\mathrm{BmN}$ cells. E. coli strains $\mathrm{DH} 5 \alpha$ and $\mathrm{DH} 10 \mathrm{~B}$ were maintained in our laboratory. BmN cells were cultured at $27^{\circ} \mathrm{C}$ in TC-100 medium supplemented with $10 \%$ Gibco fetal calf serum (Life Technologies).

\section{Transient Expression Plasmids Used for Subcellular Localization of Bm65}

Primer pair Bm65-F1 and Bm65-R was used to amplify the full length of $B m 65$, in which the TAA stop codon was deleted, and target DNA was subcloned into pFastHTB-P $\mathrm{P}_{\text {ie1-ns1-egfp }}$ to generate $\mathrm{pFastHTB}-\mathrm{P}_{\mathrm{ie} 1}-\mathrm{Bm} 65-\mathrm{egfp}$. Additionally, a series of 3'-terminally truncated Bm65 fragments were amplified from Bm-bacmid using different primer pairs as follows. Primer pair Bm65-F1 and Bm65-R1 were used to amplify Bm65(T1) with a $3^{\prime}$-terminal deletion of $60 \mathrm{bp}$; primer pair Bm65-F1 and Bm65-R2 were designed to amplify Bm65(T2) with a $3^{\prime}$-terminal deletion of 87 bp. Primer pair Bm65-F2 and Bm65-R were designed to amplify Bm65(T3) with a $5^{\prime}$-terminal deletion of 108 bp. Primer pair Bm65-F3 and Bm65-R were designed to amplify Bm65(T4) with a $5^{\prime}$-terminal deletion of 210 bp. Primer pair Bm65-F1 and Bm65-R3 were designed to amplify Bm65(T5) with a $3^{\prime}$ terminal deletion of $216 \mathrm{bp}$. The PCR products were respectively

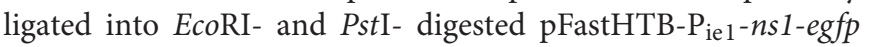
to generate the final plasmids. The final plasmids were named pFastHTB-P $\mathrm{ie}_{1-B m 65}$ (T1, T2, T3, T4, or T5)-egfp, respectively.

\section{Transient Expression of Bm65 With Mutations in ${ }^{33}$ RRIK and ${ }^{76}$ KRKCSK Motifs}

The mutation of 33R(A)34R(A)35I(A)36K(A) was introduced into Bm65 according to the instructions of the MutanBEST Kit (TaKaRa). Briefly, Bm65-F1 and Bm65-R were used to amplify Bm65 fragment for the generation of pMD18T-Bm65. Bm65M1$\mathrm{F}$ and Bm65M-R were used to amplify the $33 \mathrm{R}(\mathrm{A}) / \mathrm{Bm} 65$ from pMD18T-Bm65 to produce pMD18T-33R(A)/Bm65. In a similar way, Bm65M2-F and Bm65M-R were used to amplify 33R(A)34R(A)/Bm65 from pMD18T-33R(A)/Bm65, Bm65M3-F and Bm65M-R were used to amplify 33R(A)34R(A)35I(A)/Bm65 from pMD18T-33R(A)34R(A)/Bm65. Bm65M4-F and Bm65M$\mathrm{R}$ were used to amplify 33R(A)34R(A)35I(A)36K(A)/Bm65 from pMD18T-33R(A)34R(A)35I(A)/Bm65.

The mutation of $76 \mathrm{~K}(\mathrm{~A}) 77 \mathrm{R}(\mathrm{A}) 78 \mathrm{~K}(\mathrm{~A}) \mathrm{CS} 81 \mathrm{~K}(\mathrm{~A})$ was introduced into $\mathrm{Bm} 65$ according to the instructions of the MutanBEST Kit (TaKaRa). Firstly, Bm65M5-F and Bm65-R4 were used to amplify $76 \mathrm{~K}(\mathrm{~A}) / \mathrm{Bm} 65$ from pMD18-T-Bm65. In a similar way, Bm65M6-F and Bm65-R4 were used to amplify 76K(A)77R(A)/Bm65 from pMD18T-76K(A)/Bm65. Bm65M7-F and Bm65-R5 were used to amplify $76 \mathrm{~K}(\mathrm{~A}) 77 \mathrm{R}(\mathrm{A}) 78 \mathrm{~K}(\mathrm{~A}) / \mathrm{Bm} 65$ from pMD18T-76K(A)77R(A)/Bm65, Bm65M8-F and Bm65-R6 were used to amplify $76 \mathrm{~K}(\mathrm{~A}) 77 \mathrm{R}(\mathrm{A}) 78 \mathrm{~K}(\mathrm{~A}) 81 \mathrm{~K}(\mathrm{~A}) / \mathrm{Bm} 65$ from pMD18T-76K(A)77R(A)78K(A)/Bm65. Additionally, Bm65-F4 and Bm65-flag-R were used to amplify the cassette of $\mathrm{P}_{\mathrm{Bm} 65}$-Bm65-flag from $\mathrm{Bm}$-Bacmid, which was further mutated into $\mathrm{P}_{\mathrm{Bm} 65}-\mathrm{Bm} 65(\mathrm{M} 2)$-flag at sites $76 \mathrm{~K}(\mathrm{~A}), 77 \mathrm{R}(\mathrm{A}), 78 \mathrm{~K}(\mathrm{~A})$, and $81 \mathrm{~K}(\mathrm{~A})$ of Bm65 in a similar way. To further study the effect of single point mutation in the ${ }^{76} \mathrm{KRKCSK}$ motif on nuclear import of $\mathrm{Bm} 65,77 \mathrm{R}(\mathrm{A}), 78 \mathrm{~K}(\mathrm{~A})$, and $81 \mathrm{~K}(\mathrm{~A})$ was introduced into Bm65 by PCR amplification from pMD18T-Bm65 with 
TABLE 1 | Primers, plasmids, and viruses used in the study.

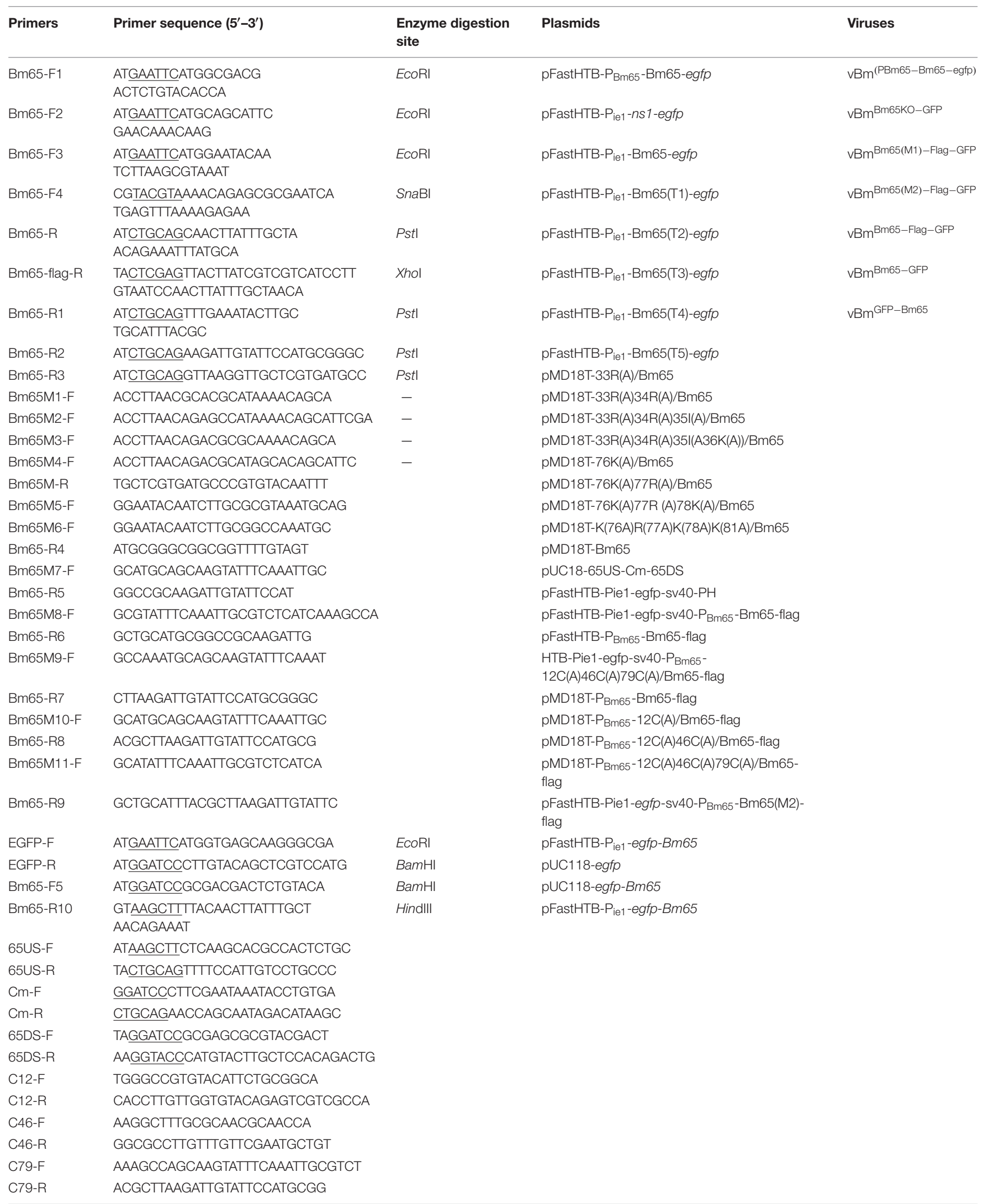

Underlined letters indicate restriction enzyme digestion sites. 
Bm65M9-F and Bm65-R7, Bm65M10-F and Bm65-R8, and Bm65M11-F and Bm65-R9, respectively.

Each mutant Bm65 sequence was isolated from the corresponding recombinant plasmid by digestion, and the resulting DNA fragments were purified and subcloned into vector pFastHTB- $\mathrm{P}_{\text {ie } 1-n s 1-e g f p}$ to generate the final plasmids. All primers used in the study are listed in Table $\mathbf{1 .}$

\section{Preparation of Recombinant Viruses}

Construction of Bm65-deleted Bm-bacmid $\left(\mathrm{Bm}^{\mathrm{Bm} 65 \mathrm{KO}}\right)$ was performed as previously described (Tang et al., 2013). Colonies resistant to chloramphenicol, ampicillin, and kanamycin were selected for PCR confirmation with 65US-F/65US-R, Cm-F/Cm$\mathrm{R}$, and 65DS-F/65DS-R (data not shown). Deletion of Bm65 and correct insertion of $\mathrm{Cm}$ cassette were named $\mathrm{Bm}^{\mathrm{Bm} 65 \mathrm{KO}}$. To generate a flag-tagged $B m 65$ repair $\mathrm{Bm}$-bacmid, the fragment containing Bm65 and its native promoter sequence tagged with flag coding sequence at the $3^{\prime}$ end was amplified from Bm-bacmid by PCR with Bm65-F4 and Bm65-flag-R. The DNA fragment was subcloned into pFastHTB-Pie1-egfp-sv40$\mathrm{PH}$ (Li et al., 2014) and pFastHTB (Invitrogen) digested with SnaBI and XhoI to generate pFastHTB-Pie1-egfp-sv40- $\mathrm{P}_{\mathrm{Bm} 65^{-}}$ Bm65-flag and pFastHTB- $\mathrm{P}_{\mathrm{Bm} 65}-\mathrm{Bm} 65$-flag, respectively. $\mathrm{P}_{\mathrm{ie} 1^{-}}$ egfp-sv40- $\mathrm{P}_{\mathrm{Bm} 65}$-Bm65-flag was transferred into the polyhedrin locus of the $\mathrm{Bm}^{\mathrm{Bm} 65 \mathrm{KO}}$, and the resulting $\mathrm{Bm}^{\mathrm{Bm}} 65-\mathrm{Flag}-\mathrm{GFP}$ was selected by blue-white screening and further confirmed by PCR with M13 primers.

Transposition between pFastHTB-Pie1-egfp-sv40-PH and $\mathrm{Bm}^{\mathrm{WT}}$ or $\mathrm{Bm}^{\mathrm{Bm} 65 \mathrm{KO}}$ was made to generate $\mathrm{Bm}{ }^{\mathrm{WT}-\mathrm{GFP}}$ and $\mathrm{Bm}^{\mathrm{Bm} 65 \mathrm{KO}-\mathrm{GFP}}$, respectively. Transposition between HTB-

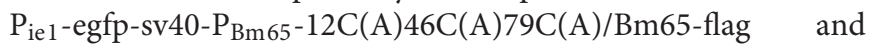
$\mathrm{Bm}^{\mathrm{Bm} 65 \mathrm{KO}}$ was made to produce $\mathrm{Bm}^{\mathrm{Bm} 65(\mathrm{M} 1)-\text { flag-GFP, }}$ which contains mutations in the three cysteine sites of Bm65. The donor plasmid of HTB-P $\mathrm{ie}_{1}$-egfp-sv40- $\mathrm{P}_{\mathrm{Bm} 65}$ $12 \mathrm{C}(\mathrm{A}) 46 \mathrm{C}(\mathrm{A}) 79 \mathrm{C}(\mathrm{A}) / \mathrm{Bm} 65$-flag was generated as follows. Briefly, $\mathrm{P}_{\mathrm{Bm} 65}-\mathrm{Bm} 65$-flag was ligated into pMD18T to generate $\mathrm{pMD} 18 \mathrm{~T}-\mathrm{P}_{\mathrm{Bm} 65}-\mathrm{Bm} 65$-flag, which was used to amplify

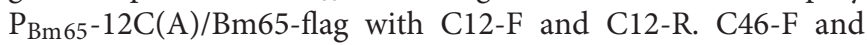
C46-R were used to amplify $\mathrm{P}_{\mathrm{Bm} 65}-12 \mathrm{C}(\mathrm{A}) 46 \mathrm{C}(\mathrm{A}) / \mathrm{Bm} 65$-flag from pMD18T- $\mathrm{P}_{\mathrm{Bm} 65}-12 \mathrm{C}(\mathrm{A}) / \mathrm{Bm} 65$-flag. Finally, $\mathrm{P}_{\mathrm{Bm} 65^{-}}$ 12C(A)46C(A)79C(A)/Bm65-flag was amplified with $\mathrm{C} 79-\mathrm{F}$ and

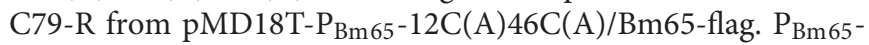
12C(A)46C(A)79C(A)/Bm65-flag was ligated into pFastHTBPiel-egfp-sv40-PH digested with SnaBI and XhoI to generate

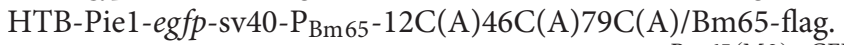

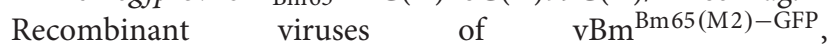

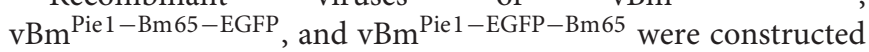
as follows. Briefly, $\mathrm{P}_{\mathrm{Bm} 65}-\mathrm{Bm} 65$ (M2)-flag was used as template for amplification with Bm65-F2 and Bm65-flag-R. $\mathrm{P}_{\mathrm{Bm}_{65}}$ Bm65(M2)-flag was ligated into pFastHTB-Pie1-egfp-sv40-PH digested with SnaBI and XhoI to generate pFastHTB-Pie1egfp-sv40-P $\mathrm{Bm}_{\mathrm{Bm}}$-Bm65(M2)-flag. pFastHTB-P $\mathrm{P}_{\text {ie } 1-B m 65-e g f p}$ was used to construct recombinant pFastHTB- $\mathrm{P}_{\text {ie } 1-e g f p-}$ Bm65. Briefly, EGFP-F and EGFP-R were used first to amplify the egfp fragment, which was subsequently ligated into pUC118 digested with EcoRI and BamHI to generate pUC118-egfp. Subsequently, Bm65-F5 and Bm65-R10 were used to amplify the Bm65 fragment, which was ligated with pUC118-egfp digested with BamHI and HindIII to generate pUC118-egfp-Bm65. Finally, the purified egfp-Bm65 from pUC118-egfp-Bm65 was ligated into pFastHTB-P $\mathrm{ie}_{1-B m 65-e g f p}$ digested with EcoRI and HindIII to generate the final pFastHTB$\mathrm{P}_{\text {ie } 1-e g f p-B m 65}$. Competent E. coli $\mathrm{DH} 10 \mathrm{~B}$ cells containing $\mathrm{Bm}^{\mathrm{Bm} 65 \mathrm{KO}}$ were transformed with pFastHTB-P $\mathrm{P}_{\text {ie }}-$ Bm65-egfp,

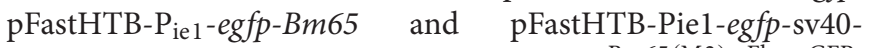

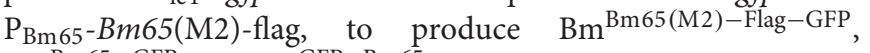
$\mathrm{Bm}^{\mathrm{Bm} 65-\mathrm{GFP}}$, and $\mathrm{Bm} \mathrm{GFP}^{\mathrm{Bm} 65}$, respectively.

\section{Transfection and Fluorescence Microscopy}

$\mathrm{BmN}$ cells $\left(10^{6}\right.$ cells/well) were seeded into six-well culture plates and incubated at $27^{\circ} \mathrm{C}$ for $16-24 \mathrm{~h}$ before transfection. Recombinant DNA molecules $(2 \mu \mathrm{g} /$ well $)$ and $5 \mu$ l Cellfectin (Invitrogen Life Technology) were mixed in $200 \mu 1 \mathrm{TC}-100$ serum free medium and incubated at $27^{\circ} \mathrm{C}$ for $45 \mathrm{~min}$. Then, $800 \mu \mathrm{l}$ serum free medium was added into the DNA-Cellfectin solution, which was finally overlaid onto $\mathrm{BmN}$ cells and incubated at $27^{\circ} \mathrm{C}$ for $5 \mathrm{~h}$. After the incubated cells were washed with serum free TC-100 medium, $2 \mathrm{ml}$ TC-100 medium containing 10\% fetal bovine serum was added into each well for further culture. At $96 \mathrm{~h}$ post-transfection (hpt), the BV-enriched culture supernatants were harvested for further study. Fluorescence in $\mathrm{BmN}$ cells was observed through fluorescence microscopy (Olympus-IX73DP80, Japan) at selected time points for further analysis. The fluorescent signal in each picture was counted one by one through observation by naked eye. The fluorescent cells were counted in each of $289 \mathrm{~mm}^{2}$ figure and 15 field of view were used for counting, which was used to make the statistical analysis using $t$-test.

\section{Western Blotting}

Recombinant viruses $\left(\mathrm{vBm}^{\mathrm{Bm}} 65-\mathrm{Flag}-\mathrm{GFP}, \quad \mathrm{vBm}\right.$

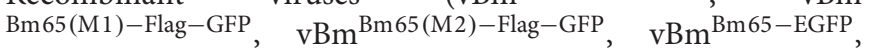
and $\left.\mathrm{vBm}^{\mathrm{EGFP}-\mathrm{Bm} 65}\right)$ were used to infect BmN cells $\left(10^{6} / 35-\mathrm{mm}\right.$ dish), which were harvested at $0,3,6,12,24,48,72$, and 96 h postinfection (hpi). The cell pellets were respectively resuspended in $200 \mu \mathrm{l}$ of RIPA Lysis Buffer (50 mM Tris [pH 7.4], $150 \mathrm{mM}$ $\mathrm{NaCl}, 1 \%$ Triton $\mathrm{X}-100,1 \%$ sodium deoxycholate, $0.1 \%$ SDS [Beyotime]), and protein concentrations of cell lysates were determined with BCA Protein Assay kit (Pierce) according to the manufacturer's instructions. Total protein $(30 \mu \mathrm{g})$ in each sample was dissolved in sample buffer (10 mM Tris- $\mathrm{HCl} \mathrm{pH} \mathrm{8.0,1 \%} \mathrm{SDS,}$ $10 \%$ Glycerol, $0.008 \%$ Bromophenol Blue) with or without 5\% (v/v) $\beta$-mercaptoethanol, which was subjected to SDS-PAGE and further transferred onto polyvinyldifluorene (PVDF) membranes (Millipore) as previously described (Yao et al., 2019).

The blots were blocked with $5 \%$ non-fat milk in $1 \times$ PBST (2.6 mM KCl, 0.136M NaCl, $8 \mathrm{mM} \mathrm{Na} \mathrm{HPO}_{4}, 2 \mathrm{mM}$ $\mathrm{KH}_{2} \mathrm{PO}_{4}, 0.1 \%$ Tween $20, \mathrm{pH} 7.4$ ) overnight at $4^{\circ} \mathrm{C}$ and then incubated with antibodies against flag tag (Code\#HT201, TransGen Biotech) at a dilution of 1:2000 in 5\% non-fat milk in $1 \times$ PBST. After incubation with primary antibody, the blots were washed with $1 \times$ PBST for $10 \mathrm{~min}$, which was 
repeated three times. Subsequently, the blots were incubated with goat anti-rat IgG conjugated to horseradish peroxidase (HS201-01, TransGen Biotech) diluted 1:5000 for $1 \mathrm{~h}$ at room temperature. The hybridization signal was visualized using enhanced chemiluminescence (ECL) (Amersham).

\section{Confocal Microscopy}

Confocal microscopy was performed as previously described (Li et al., 2015; Tang et al., 2015), with some modifications. Briefly, BmN cells $\left(1 \times 10^{5}\right)$ were seeded into a 35$\mathrm{mm}$ glass-bottom cell culture dish (NSET). The cells were transfected with $2 \mu \mathrm{g}$ recombinant plasmids, which can transiently express Bm65 and Bm65 truncations fused with EGFP under the control of ie1 promoter. The cell culture supernatants were removed at $48 \mathrm{hpt}$ and the cells were used for subcellular localization analysis of Bm65. Additionally, $\mathrm{BmN}$ cells $\left(1 \times 10^{5}\right)$ infected with $\mathrm{vBm}^{\mathrm{Bm} 65(\mathrm{M} 2)-\mathrm{GFP}}$ and $\mathrm{vBm}^{\mathrm{Bm} 65-\mathrm{GFP}}$ were also used to study the subcellular localization of Bm65. The cell culture supernatants were removed at 3, $6,12,24,48$, and $72 \mathrm{hpi}$, respectively. Subsequently, these $\mathrm{BmN}$ cells were washed with PBS $(2.6 \mathrm{mM} \mathrm{KCl}, 0.136 \mathrm{M}$ $\mathrm{NaCl}, 8 \mathrm{mM} \mathrm{Na} \mathrm{HPO}_{4}, 2 \mathrm{mM} \mathrm{KH} \mathrm{PO}_{4}, \mathrm{pH}$ 7.4), fixed with $4 \%$ paraformaldehyde for $15 \mathrm{~min}$, washed three times with PBS for $10 \mathrm{~min}$, and permeabilized in $0.1 \%$ Triton $\mathrm{X}$ 100 for $15 \mathrm{~min}$. Finally, the cells were stained with DAPI (60 $\mu \mathrm{g} / \mathrm{ml}$, Sigma) for $10 \mathrm{~min}$, and washed three times with PBS. In addition, $\mathrm{BmN}$ cells $\left(1 \times 10^{5}\right)$ infected with $\mathrm{vBm}^{\mathrm{Bm} 65(\mathrm{M} 2)-\mathrm{Flag}-\mathrm{GFP}}$ and $\mathrm{vBm}^{\mathrm{Bm} 65-\mathrm{Flag}-\mathrm{GFP}}$ were used for subcellular localization of Bm65 by immunocytochemistry assay. Briefly, BmN cells were treated with monoclonal antibodies (TransGen Biotech) against Flag, followed by treatment with secondary antibody conjugated-FITC (Alexa Fluor $647^{\circledR}$, ab150107) and the nucleus was treated with DAPI (60 $\mu \mathrm{g} / \mathrm{ml}$, Sigma).

The cells were directly observed and photographed using Confocal Laser Scanning Microscopy (Model:TCS SP5 II, Leica corporation of Germany). Laser source is single-laser excitation source for multicolor and fluorescence emission was excited at 488 and $345 \mathrm{~nm}$ to detect the fluorescence signal of EGFP and DAPI, respectively. Glycerol objective $(\times 63)$ was used for imaging.

\section{Analysis of Viral Growth Curve}

To further study whether deletion of Bm65 and alanine mutations in the ${ }^{76}$ KRKCSK motif affected production of recombinant virus, a virus growth curve analysis was performed as described previously (Tang et al., 2013). A monolayer of $\mathrm{BmN}$ cells $\left(10^{6}\right)$ was seeded in six-well plates for transfection with $2.0 \mu \mathrm{g}$ of bacmid DNA and the experiments were repeated three times. The supernatants of transfected cells containing recombinant viruses were harvested at selected time points. The $50 \%$ tissue culture infective dose $\left(\mathrm{TCID}_{50}\right)$ end-point dilution was carried out to determine the titer of $\mathrm{BV}$ as described previously, with modifications (O'Reilly et al., 1994). Viral infections were characterized by observation of fluorescence in $\mathrm{BmN}$ cells. It was identified to be positive if green fluorescence was observed in one or more BmN cells by fluorescence microscopy. Statistical analysis was performed using single factor analysis of variance.

\section{RESULTS}

\section{Comparison of the Amino Acid Sequences of Bm65 Homologs}

Bm65 codes for 104 deduced amino acid residues with a putative molecular mass of $12.2 \mathrm{kDa}$. Bm65 is not a core gene existing in all baculoviruses. Alignment result revealed that it is a highly conserved gene in baculoviruses of the genera Alphabaculovirus and Betabaculovirus (Figure 1). Ac79 [orf79 of Autographa californica multiple nucleopolyhedrovirus (AcMNPV)], a homologs of Bm65, has been reported to be an early gene involved in efficient budded virus production (Wu and Passarelli, 2012). Tang et al. (2015) reported that a conserved motif of GIY-YIG nuclease superfamily was found in the sequence of Bm65. Moreover, two possible typical nuclear localization signal (NLS) motifs of ${ }^{33}$ RRIK and ${ }^{76}$ KRKCSK are contained in the middle part of Bm65 sequence, and alignment result revealed that they are conserved in the Bm65 sequence and its homologs, Additionally, a leucine-rich motif of ${ }^{92}$ PLLLHKFLL in the C-terminal part of Bm65 sequence was found to be conserved in the multiple sequences, which was predicted to be a potential nuclear export signal by analysis of the online tool ${ }^{1}$.

\section{Analysis of Recombinant Bm65 Expressed in BmNPV-Infected BmN Cells}

Construction of recombinant virus for expression of Bm65 is described as Figure 2A. Flag-tagged Bm65 was examined from 3 to 72 hpi under the control of its natural promoter (Figures 2BD). Western blotting analysis of total protein from $\mathrm{BmN}$ cells infected with $\mathrm{vBm}^{\mathrm{Bm} 65-\text { flag-GFP }}$ or $\mathrm{vBm}^{\mathrm{Bm} 65(\mathrm{M} 1)-F l a g-G F P}$ was carried out. A specific protein band was examined from 3 to 72 hpi (Figure 2B). However, the experimentally determined mass of Bm65 was about four times as large as that of the predicted size of Bm65, indicating that Bm65 exists with a mainly tetrameric form in BmNPV-infected BmN cells. Protein lysate from BmN cells infected with vBm-bacmid ${ }^{\text {Bm65-Flag-GFP }}$ was treated with $\beta$-mercaptoethanol, which was subsequently subjected to Western blotting analysis. Only a Bm65 specific protein band migrating at only a size of approx $12 \mathrm{kDa}$ was observed from 3 to 72 hpi (Figure 2C), showing that treatment of $\beta$-mercaptoethanol transformed the tetrameric form of Bm65 into a monomer. To explore the formative mechanism of Bm65 tetramer, three cysteine residues at positions 12,46 , and 79 of Bm65 were substituted by alanine residues and the mutant name fusion with flag was defined as Bm65(M1)-flag. The total protein from $\mathrm{BmN}$ cells infected with recombinant virus for expression of Bm65 mutant was determined by Western blotting. Only the tetrameric form of Bm65 was detected from 3 to 96 hpi (Figure 2D), indicating that the mutations did not

${ }^{1}$ http://www.cbs.dtu.dk/services/NetNES/ 


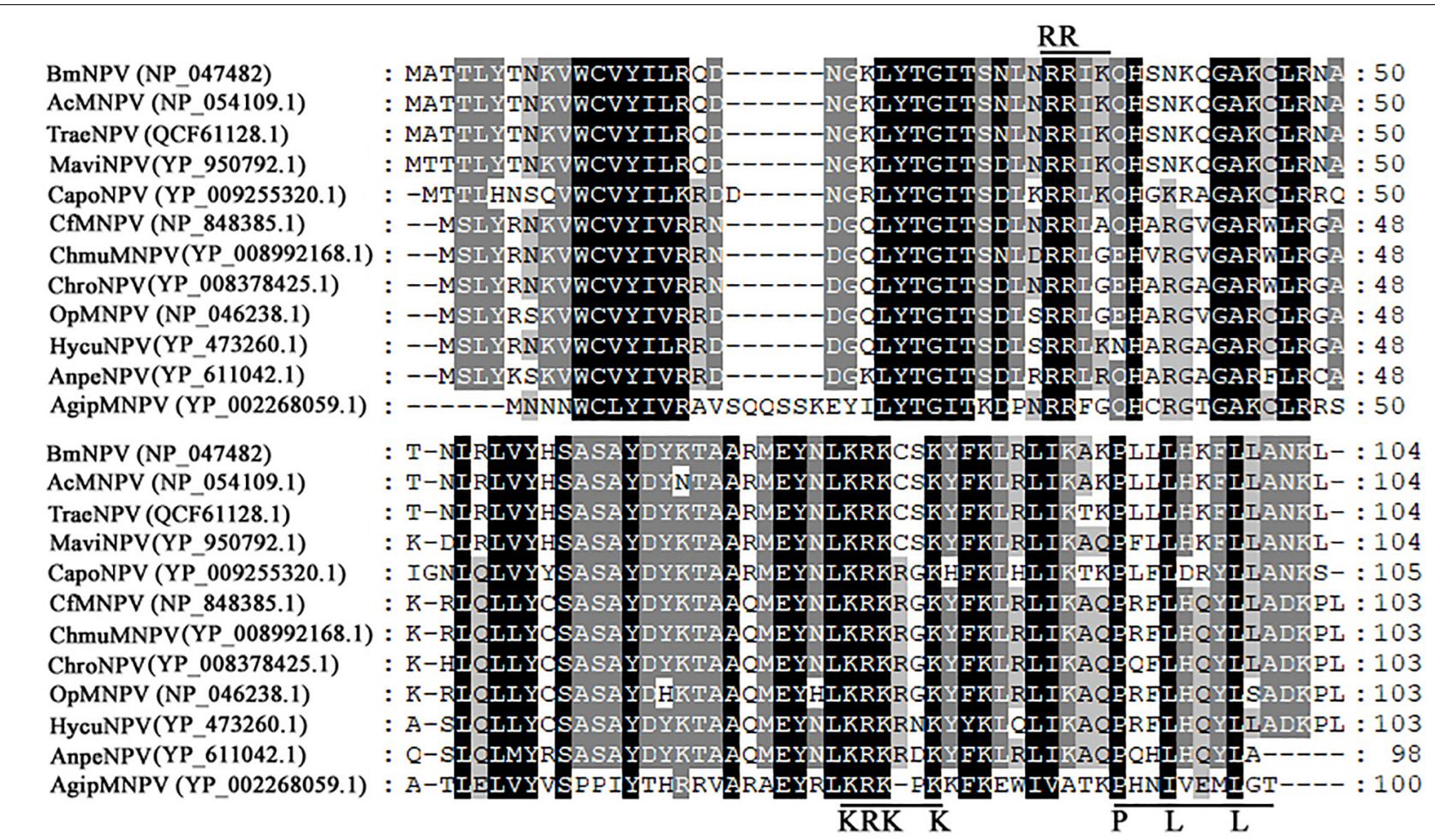

FIGURE 1 | Alignment of Bm65 and its homologs. The alignment was performed using Clustal W and edited using Genedoc software. Identical amino acids are denoted by black shading and similar amino acids are denoted by gray shading. These sequences are from GenBank, and the accession numbers are as follows: BmNPV (NP_047482.1), AcMNPV (NP_054109.1), TraeNPV (QCF61128.1), MaviNPV (YP_950792.1), CfMNPV (YP_950792.1), ChmuMNPV (YP_008992168.1), ChroNPV (YP_008378425.1), OpMNPV (NP_046238.1), HycuNPV (YP_473260.1), AnpeNPV (YP_611042.1), CapoNPV (YP_009255320.1), and AgipMNPV (YP_002268059.1).

prevent the formation of Bm65 tetramer. Additionally, extrabands of about $26 \mathrm{kDa}$ appear in Panel D from 48 to 72 hpi. They may be Bm65 dimer or Bm65 protein complex. Further experiments would be required to characterize this $26 \mathrm{kDa}$ band. In conclusion, these results revealed that Bm65 is an early protein with a tetrameric form existing in BmNPVinfected $\mathrm{BmN}$ cells, but the formative mechanism requires further research.

\section{Subcellular Localization of Bm65 Truncations Fusion With EGFP}

Bm65 is an early protein correlated with the repair of damaged DNA in nucleus of host cell (Tang et al., 2015, 2017). Therefore, it is indispensable for Bm65 to enter the nucleus of target cell for DNA repair. However, the mechanism of $\mathrm{Bm} 65$ translocation into nucleus remains unknown.

Two possible NLS motifs of ${ }^{33}$ RRIK and ${ }^{76}$ KRKCSK, and a possible nuclear export signal of ${ }^{92}$ PLLLHKFLL are contained in the Bm65 sequence. To identify whether these putative motifs are functional, four truncations with the C-terminal and N-terminal deletions of Bm65 fusion with EGFP under control of ie1 promoter were constructed to study intracellular distribution of Bm65, by observation of fluorescence signal. $\mathrm{BmN}$ cells were not infected in this assay. Transient expression assay showed that Bm65 was located mainly in the nuclei and only a little in the cytoplasm, but the intracellular distribution of truncated forms of Bm65 was correlated with the deletions of Bm65 (Figure 3). The truncated form of Bm65 (aa 1-84) accumulated almost exclusively in the nuclei of $\mathrm{BmN}$ cells. Compared with the truncated form of Bm65 (aa 1-84), a small part of green fluorescence was found in the cytoplasm except for the majority of green fluorescence accumulated in nucleus from the panel 2 of Figure 3 when $\mathrm{BmN}$ cells were transfected with the complete Bm65. The result indicated that the ${ }^{92}$ PLLLHKFLL motif may be involved with nuclear export of Bm65. The motif of ${ }^{92}$ PLLLHKFLL may be a functional nuclear export signal for transport of Bm65 from nucleus to cytoplasm. Further research is required to demonstrate the role of the ${ }^{92}$ PLLLHKFLL motif on nuclear export of Bm65. However, the truncated form (aa 1-75) of Bm65 was uniformly distributed within $\mathrm{BmN}$ cells when Bm65 with the C-terminal deletion of 29 aa, containing the motifs of ${ }^{92}$ PLLLHKFLL and ${ }^{76}$ KRKCSK, was expressed in $\mathrm{BmN}$ cells, indicating that ${ }^{76} \mathrm{KRKCSK}$ may be a functional NLS for the active transport of Bm65 from cytoplasm to nucleus. Additionally, the truncated forms (aa 37-104 and aa 71-104) of Bm65 were accumulated mainly in the nucleus and only a little in the cytoplasm. The truncated form (aa 1-36) of Bm65 was evenly distributed in 
A

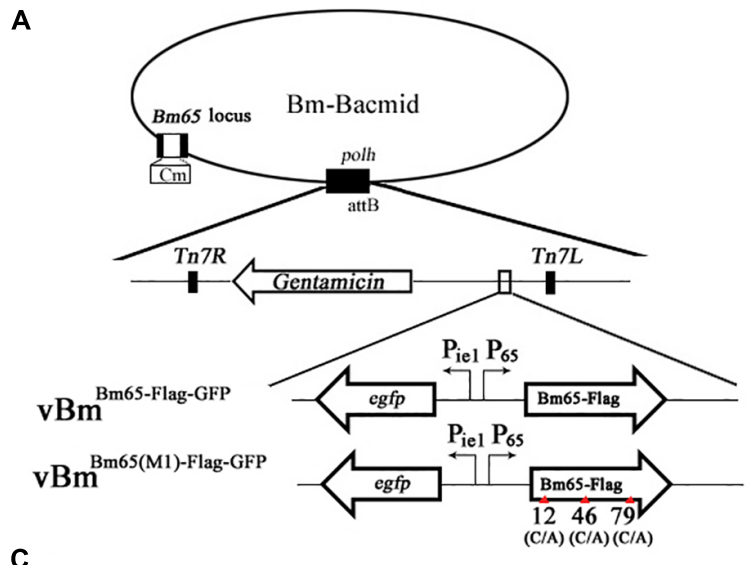

c

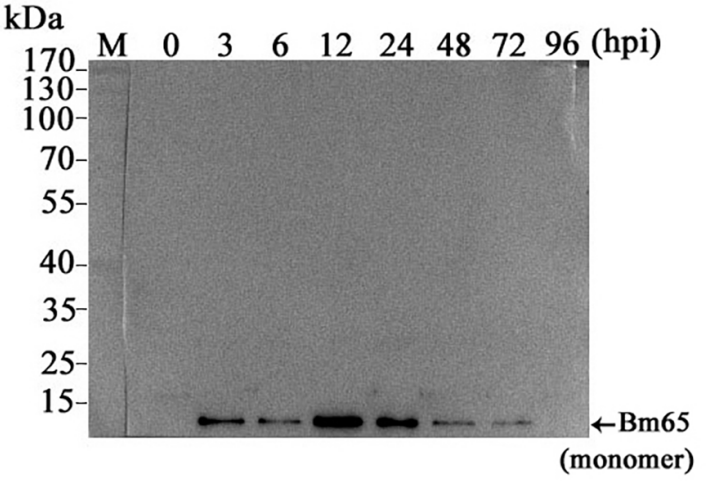

B

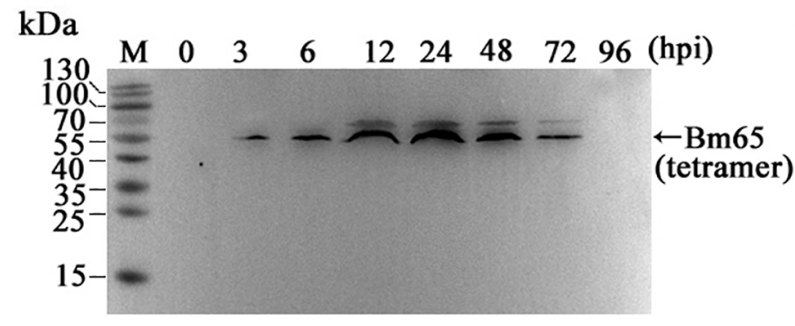

D

$\mathrm{kDa}$

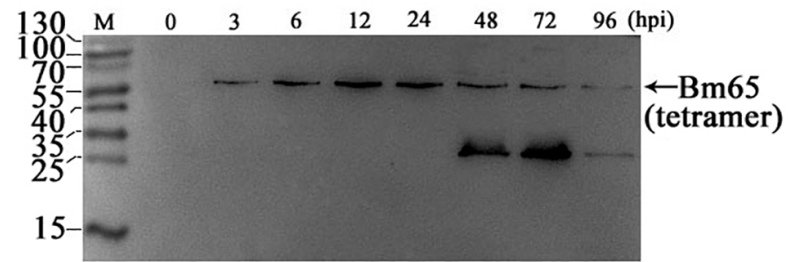

FIGURE 2 | Western blotting analysis of Bm65-flag and Bm65(M1)-flag using antibodies against flag (Code\#HT201 TransGen Biotech). (A) Strategy for expression of recombinant protein Bm65-flag and Bm65(M1)-flag. (B) Western blotting analysis of Bm65-flag expressed in BmNPV-infected BmN cells. (C) Western blotting analysis of Bm65-flag expressed in virus-infected BmN cells treated with $\beta$-mercaptoethanol. (D) Western blotting analysis of Bm65(M1)-flag expressed in virus-infected BmN cells. The prestained protein standards are on the left. The virus of vBm ${ }^{B m 65(M 1)-F l a g-G F P}$ used is one in which all three cysteines were changed to alanine.

transfected $\mathrm{BmN}$ cells. The results indicated that the motif of ${ }^{33}$ RRIK has no effect on the nuclear import of Bm65, and the nuclear import of Bm65 is strictly dependent of the ${ }^{76}$ KRKCSK motif.

\section{Effect of Alanine Mutations in the Motifs of ${ }^{33}$ RRIK and ${ }^{76}$ KRKCSK on the Nuclear Import of $\mathrm{Bm} 65$}

The truncated form (aa 37-104) of Bm65 with deletion of the ${ }^{33}$ RRIK motif was found to be accumulated mainly in the nucleus, which is in line with the subcellular localization of Bm65 in BmN cells. The result indicated that the deletion did not affect the trafficking of Bm65 from cytoplasm to nucleus. To confirm this, alanine mutations were introduced into the ${ }^{33}$ RRIK motif of Bm65 to study the subcellular distribution of Bm65 through fluorescence observation. The ${ }^{33} \mathrm{AAAA}$ (mutant 1) was accumulated mainly in the nucleus and some fluorescent aggregates were also present (Figure 4). However, the ${ }^{76}$ AAACSA (mutant 2) altered the pattern of subcellular localization of Bm65 in BmN cells, and the mutations severely blocked the transport of Bm65 from cytoplasm to nucleus. Fluorescence was accumulated exclusively in cytoplasm, and there were some fluorescent aggregates in the cytoplasm (Figure 4). According to the above results, it is believed that the motif of ${ }^{33}$ RRIK is not involved with the nuclear import of Bm65, but the motif of ${ }^{76} \mathrm{KRKCSK}$ is an efficient NLS for transport of Bm65 from cytoplasm to nucleus.

To define the importance of basic amino acid residues in the motif of ${ }^{76} \mathrm{KRKCSK}$, a series of single point mutations, including ${ }^{76} \mathrm{ARKCSK}$ (mutant 3 ), ${ }^{76} \mathrm{KAKCSK}$ (mutant 4), ${ }^{76}$ KRACSK (mutant 5) and ${ }^{76} \mathrm{KRKCSA}$ (mutant 6), were made to examine the subcellular localization of Bm65 by fluorescence microscopy. Mutant 3 did not block the nuclear import of Bm65, but other mutations impaired or blocked the nuclear import of Bm65 (Figure 3). Mutant 4 obviously impaired the nuclear import of Bm65, because some fluorescence was accumulated in the cytoplasm of some cells, and some fluorescence was found to be only in nucleus of some cells. The fluorescence of typically accumulated in cytoplasm for the mutation of mutant 4 was selected to describe the phenomenon of reduced nuclear entry (Figure 4). The result indicated that the residue ${ }^{77} \mathrm{R}$ is involved with the efficiency of nuclear import. Mutant 5 and mutant 6 severely blocked the nuclear import of Bm65, and all fluorescence was accumulated dominantly in the cytoplasm. 


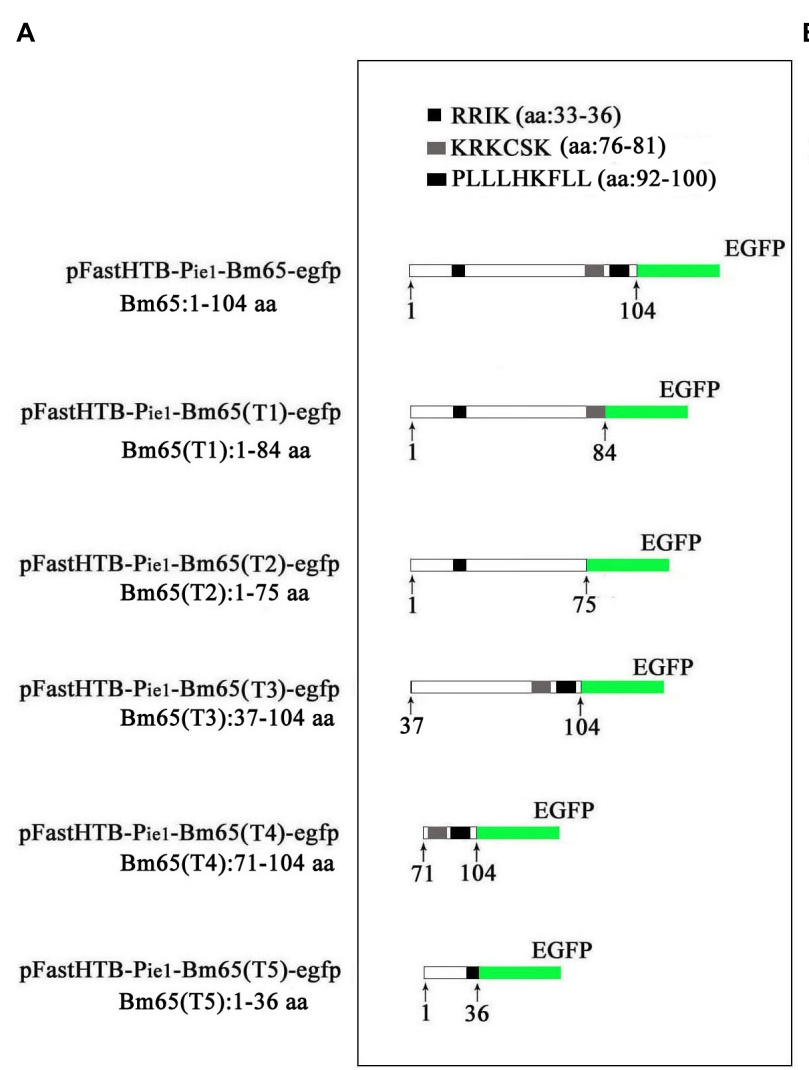

B

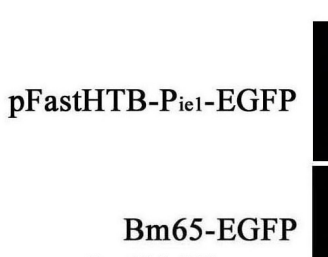

Bm65:1-104 aa

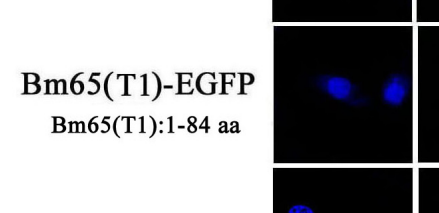

Bm65(T2)-EGFP

Bm65(T2):1-75 aa

Bm65(T3)-EGFP

Bm65(T3):37-104 aa

Bm65(T4)-EGFP
Bm65(T4):71-104 aa

Bm65(T5)-EGFP

Bm65(T5):1-36 aa

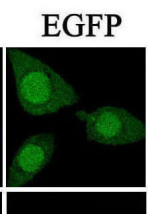

Merge
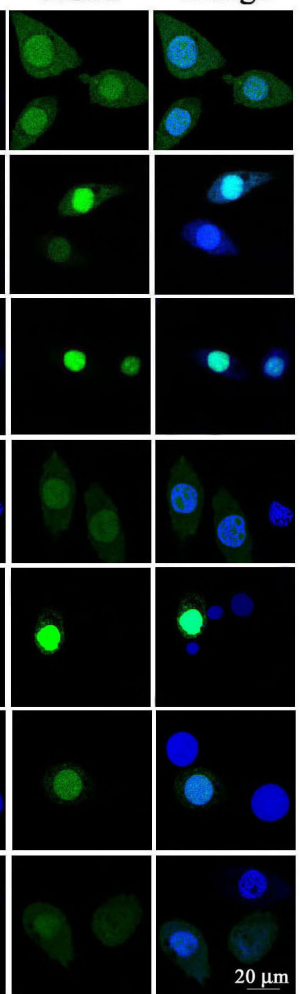

FIGURE 3 | Confocal microscopy of truncated Bm65 fused with EGFP expressed in BmN cells. (A) Strategy for the construction of truncated Bm65 fusion with the N-terminal EGFP. Black boxes correspond to the basic residue cluster ${ }^{33}$ RRIK and a leucine-rich region ${ }^{92} \mathrm{PLLLHKFLL}$. Gray box and green boxes correspond to

${ }^{76}$ KRKCSK and EGFP, respectively. (B) Fluorescence microscopy of truncated regions of Bm65 fusion with EGFP expressed in BmN cells. The transient expression

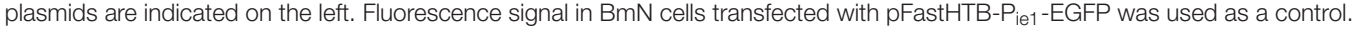

In conclusion, these mutations resulted in the varied distribution pattern of green fluorescence in $\mathrm{BmN}$ cells compared with that of wild type Bm65. The intracellular distribution of fluorescence is summarized in Table 2. The results indicated that the ${ }^{76}$ KRKCSK motif is an efficient NLS for the final destination of $\mathrm{Bm} 65$, and the residues of ${ }^{78} \mathrm{~K}$ and ${ }^{81} \mathrm{~K}$ are essential for nuclear import of Bm65.

\section{The ${ }^{76}$ AAACSA Mutant Blocked the Nuclear Import of Bm65}

To further study the effect of the mutant 2 on the subcellular localization of $\mathrm{Bm} 65$ in virus-infected $\mathrm{BmN}$ cells, recombinant virus of $\mathrm{vBm}^{\mathrm{Bm} 65(\mathrm{M} 2)-\mathrm{GFP}}$ (Figure 5A) was used to infect $\mathrm{BmN}$ cells for expression of mutant Bm65 EGFP fusion. Bm65 labeled EGFP was under the control of ie1 promoter. The fluorescence signal was observed exclusively in the cytoplasm of virus-infected $\mathrm{BmN}$ cells at $3 \mathrm{hpi}$, and the fluorescence continued to be observed in cytoplasm from 6 to $72 \mathrm{hpi}$. Additionally, Bm65(M2)-GFP showed fluorescent aggregates in the form of punctate dots in the cytoplasm of virusinfected cells (Figure 5B). Together, these results demonstrated that alanine mutations including K76A, R77A, K78A, and K81A completely blocked the nuclear import of Bm65 in virus-infected $\mathrm{BmN}$ cells, and the motif of ${ }^{76} \mathrm{KKKCSK}$ was confirmed to be an efficient NLS for transport of Bm65 from cytoplasm to nucleus.

$\mathrm{vBm}^{\mathrm{Bm} 65-\mathrm{GFP}}$ was used as the control of wild type (Figure 5C). The result indicated that Bm65-GFP was accumulated mainly in the nucleus of virus-infected $\mathrm{BmN}$ cells, and some fulorescent aggregates were found to be accumulated in nucleus of virus-infected $\mathrm{BmN}$ cells (Figure 5D). Additionally, Tang et al. (2015) have examined the subcellular localization of wild type Bm65-EGFP by the observation of confocal microscopy. The results also confirmed that fluorescence signal and some fluorescenct aggregates were accumulated mainly in nucleus of virusinfected $\mathrm{BmN}$ cells. Compared with the localization of wild type Bm65, the mutant 2 was accumulated mainly in cytoplasm of virus-infected $\mathrm{BmN}$ cells. The results indicated that the motif of ${ }^{76} \mathrm{KKKCSK}$ was an efficient NLS for the nuclear import of Bm65.

\section{Disruption of Intrinsic Stability of the Tetramer for Bm65 Fusion With EGFP}

Due to some mutations in the ${ }^{76}$ KRKCSK NLS, Bm65 mutants were found to be accumulated exclusively in cytoplasm. 
A

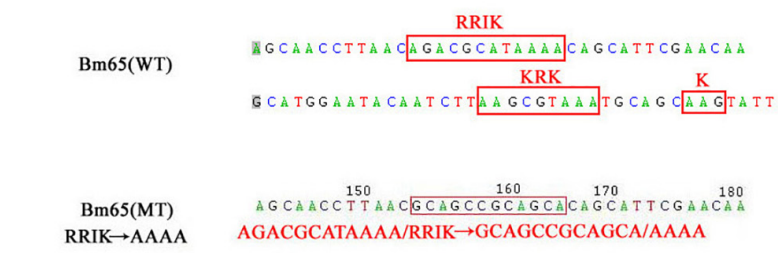

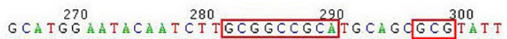
$\mathrm{KRKCSK} \rightarrow$ AAACSA $\mathrm{AAGCGTAAA} / \mathrm{KRK} \rightarrow$ GCGGCCGCA/AAA $\mathrm{AAG} / \mathrm{K} \rightarrow \mathrm{GCG} / \mathrm{A}$

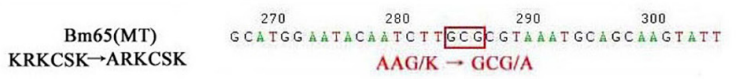

Bm65(MT)
KRKCSK $\rightarrow$ KAKCSK 270
GCATG G AATACAATCTTAAGGCOA A ATGCAGCAAGTATT
CGT/R $\rightarrow$ GCC/A (

Bm65(MT)

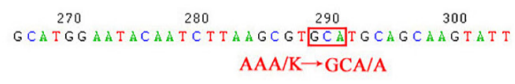

Bm65(MT)
KRKCSK $\rightarrow$ KRKCSA

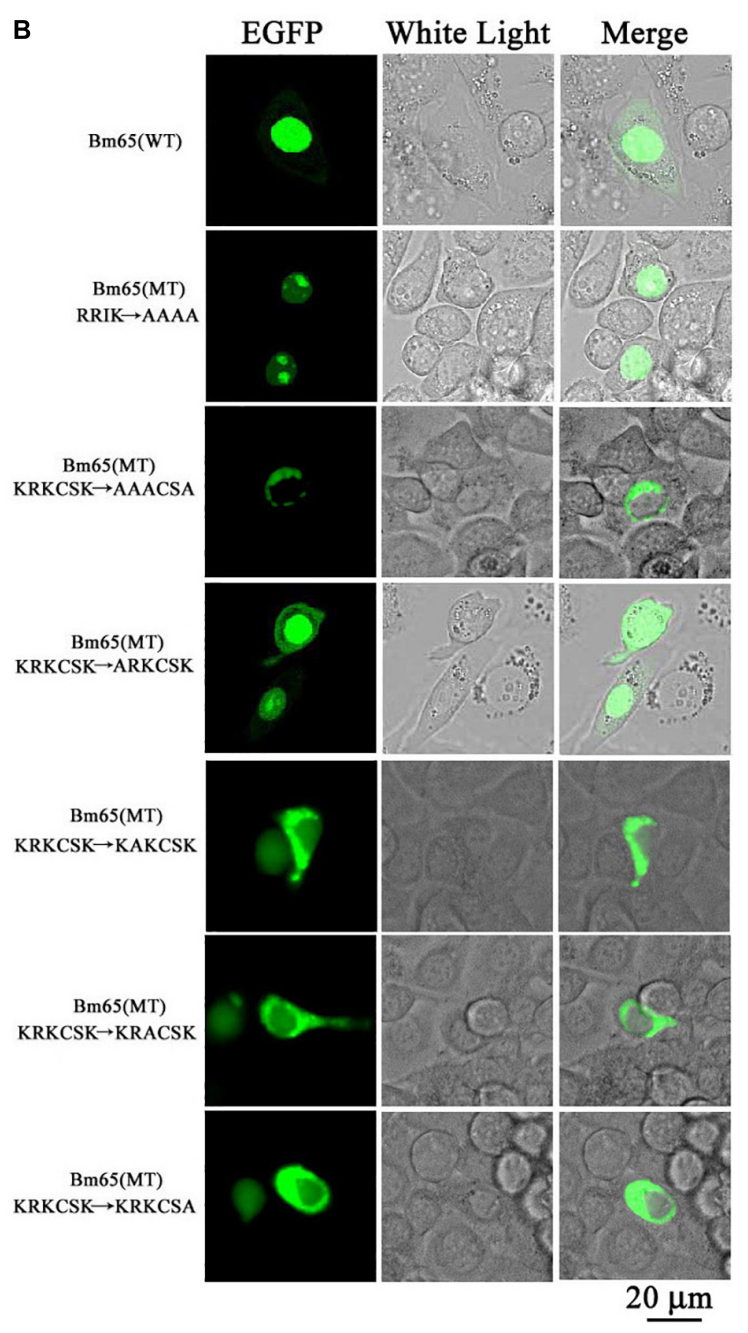

FIGURE 4 | Effect of mutations on the subcellular localization of Bm65 expressed in BmN cells. (A) Strategy for construction of a series of transient expression vectors for expression of Bm65 mutant fusion with EGFP. (B) Intracellular distribution of green fluorescence in BmN cells transfected with a series of transient expression vectors. Plasmids used for transfection are indicated on the left. The numbers relative to the nucleotides position in DNA sequence were indicated above the sequence. The mutated codons in the sequence of Bm65 were confirmed by sequencing and enclosed in red letters. Alanine mutations in the motifs of ${ }^{33} \mathrm{RRIK}$ and ${ }^{76}$ KRKCSK are indicated below the sequence. MT, mutant type; WT, wild type.

To determine the effect of alanine mutations in the motif ${ }^{76} \mathrm{KRKCSK}$ on the tetrameric form of Bm65, recombinant virus $\mathrm{vBm}^{\mathrm{Bm}} 65(\mathrm{M} 2)-\mathrm{Flag}-\mathrm{GFP}$ (Figure 6A) for expression of Bm65 mutant was made and used to infect $\mathrm{BmN}$ cells for Western blot analysis. Only a tetramer specific band of Bm65 was detected from 24 to 72 hpi (Figure 6B), and alanine mutations in the motif ${ }^{76} \mathrm{KRKCSK}$ did not affect the formation of Bm65 tetramer. So, the tetrameric form of Bm65 can be produced in cytoplasmic and nuclear sites, but it was not required for the nuclear import of Bm65 because the monomer of Bm65-GFP also entered nucleus. According to the above results, it is regarded that the introduced mutations were not involved with the tetramer formation and/or on protein stability.

To further determine whether a fusion protein labeled with $\mathrm{Bm} 65$ produced the tetrameric form in $\mathrm{BmN}$ cells, recombinant viruses for expression of the $\mathrm{N}$-terminal or C-terminal extension of Bm65 labeled with EGFP was under the control of ie1 promoter, which were prepared and used to infect $\mathrm{BmN}$ cells for Western blotting analysis. A band migrating at $40 \mathrm{kDa}$, the size expected for a Bm65:EGFP fusion was detected from 24 to 72 hpi (Figures 6C,D) using antibodies against EGFP, and only the monomer form of Bm65 fusion, regardless of N-terminal or C-terminal, was formed. According to the result, it is regarded that EGFP fusion affect the stability the tetramer of Bm65.

\section{Immunocytochemistry Assay for Distribution of Bm65-Flag and Bm65(M2)-Flag}

Although GFP-tagged Bm65 and its variants are sufficient to just prove nuclear localization ability of the NLS, Western 
TABLE 2 | Effect of ${ }^{33}$ RRIK and ${ }^{76}$ KRKCSK motifs on nuclear accumulation of Bm65.

\begin{tabular}{lll}
\hline Mutants & $\begin{array}{l}\text { Sequence and } \\
\text { mutations }\end{array}$ & $\begin{array}{l}\text { Subcellular } \\
\text { localization }\end{array}$ \\
\hline WT & ${ }^{33} \mathrm{RRIK},{ }^{76} \mathrm{KRKCSK}$ & $\mathrm{N}(++) / \mathrm{C}$ \\
Truncation & Bm65 (aa 37-104) & $\mathrm{N}(++) / \mathrm{C} \mathrm{N}(++) / \mathrm{C} \mathrm{N} / \mathrm{C}$ \\
Truncation & Bm65 (aa 1-84) Bm65 & \\
Truncation & $($ aa 1-75) & \\
Mutant 1 & ${ }^{33}$ AAAA & $\mathrm{N}(++) / \mathrm{C}$ \\
Mutant 2 & ${ }^{76}$ AAACSA & $\mathrm{C}$ \\
Mutant 3 & ${ }^{76}$ ARKCSK & $\mathrm{N}(+) / \mathrm{C}$ \\
Mutant 4 & ${ }^{76}$ KAKCSK & $\mathrm{N}(+) / \mathrm{C}$ \\
Mutant 5 & ${ }^{76}$ KRACSK & $\mathrm{C}$ \\
Mutant 6 & 76 KRKCSA & $\mathrm{C}$ \\
\hline
\end{tabular}

$N(++) / C, N(+) / C, N / C$, and $C$ indicated that fluorescence signal was predominantly but not mainly in the nucleus, in the nucleus was heavier than that of in the cytoplasm, equally between the nucleus and the cytoplasm, and mainly in the cytoplasm (C), respectively.

blotting revealed that the recombinant virus expressing Bm65GFP also expressed a large amount of extra proteins detected by anti-GFP antibody (Figures $6 \mathbf{C , D}$ ). To preclude the possibility of fluorescent aggregates from the huge amount of truncated GFP and the mislocalization of Bm65 caused by GFP-tag, the immunocytochemistry assay was made in $\mathrm{vBm}^{\mathrm{Bm} 65(\mathrm{M} 2)-\mathrm{Flag}-\mathrm{GFP}}$-infected $\mathrm{BmN}$ cells using antibodies against flag tag (Figure 7C). Meanwhile, vBm ${ }^{\text {Bm65-Flag-GFP }}$ was used as a control (Figure 7A). The immunocytochemistry assay of Bm65-flag was made in $\mathrm{vBm}^{\mathrm{Bm} 65-\mathrm{Flag}-\mathrm{GFP}}$-infected $\mathrm{BmN}$ cells using antibodies against flag, which was used as a control of wild type. The subcellular localization of Bm65 and its variant was judged from the distribution of red fluorescent signal observed by confocal microscopy.

The results indicated that Bm65-flag was accumulated mainly in the nucleus of BmNPV-infected $\mathrm{BmN}$ cells, and some fluorescent aggregates were found to be accumulated in nucleus of virus-infected BmN cells (Figure 7B). However, the mutant 2 was accumulated mainly in cytoplasm of virus-infected $\mathrm{BmN}$ cells and some fluorescent aggregates was found to be accumulated in cytoplasm (Figure 7D).

\section{Alanine Mutations in the Motif ${ }^{76}$ KRKCSK Sharply Impaired the Production of Infectious Virions}

To analyze whether Bm65 is required for viral production, four recombinant $\mathrm{Bm}$-Bacmids, including fully deleted-type $\left(\mathrm{Bm}^{\mathrm{Bm} 65 \mathrm{KO}-\mathrm{GFP}}\right)$, wild-type $\left(\mathrm{Bm} \mathrm{WT}^{\mathrm{WFP}}\right)$, repair-type 1 $\left(\mathrm{Bm}^{\mathrm{Bm} 65-\text { Flag-GFP }}\right)$, and repair-type $2\left(\mathrm{Bm}^{\mathrm{Bm} 65(\mathrm{M} 2)-\mathrm{Flag}-\mathrm{GFP}}\right)$ were generated and further transfected into $\mathrm{BmN}$ cells. Green fluorescence was observed by fluorescence microscopy in individual cultured cells as early as $24 \mathrm{hpt}$. However, in cells

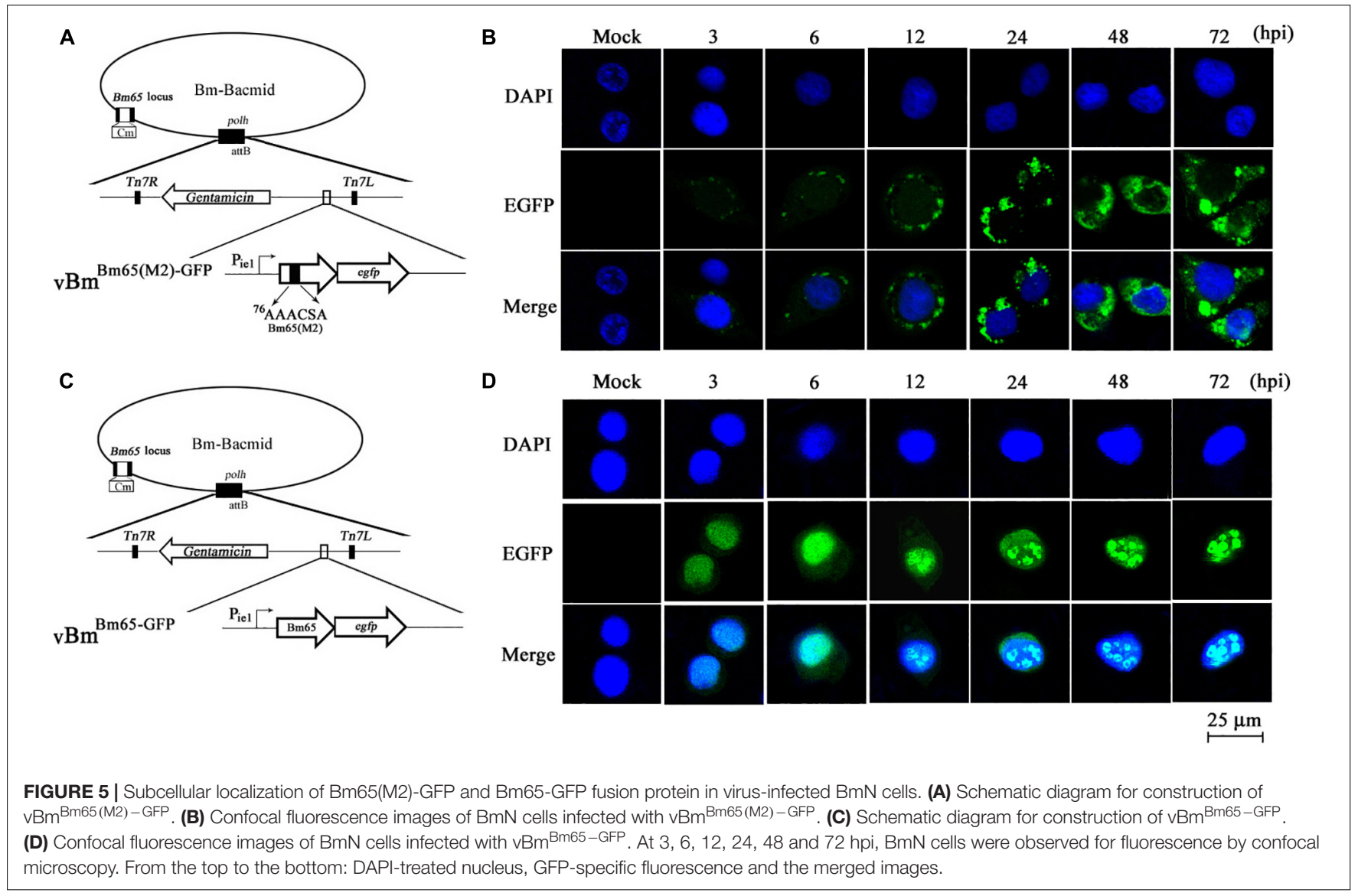


A

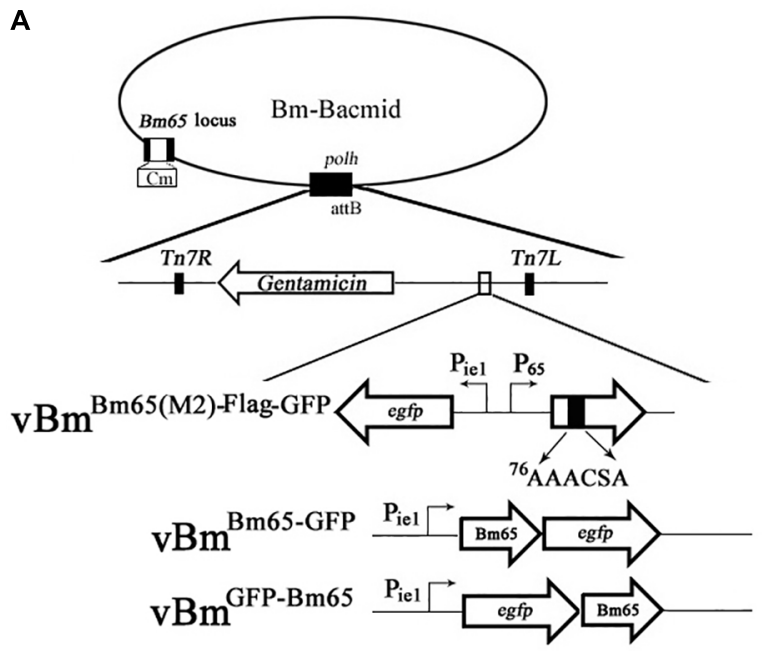

C

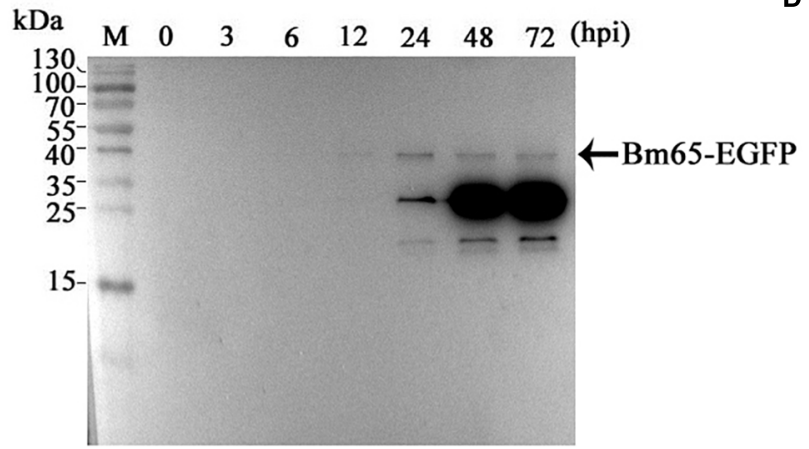

B

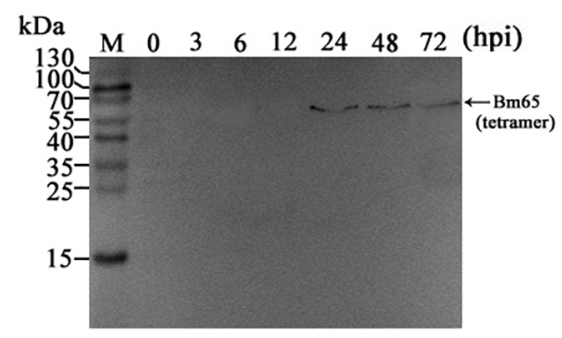

D

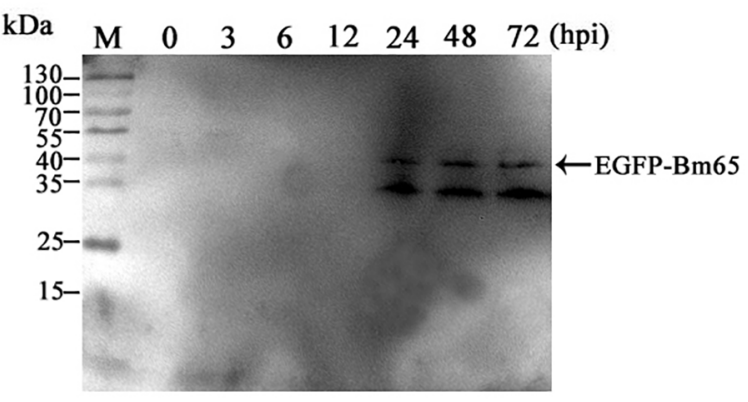

FIGURE 6 | Effect of alanine mutations in the motif ${ }^{76} \mathrm{KRKCSK}$ and Bm65 labeled with EGFP on Bm65 tetramer. (A) Strategy for construction of three recombinant

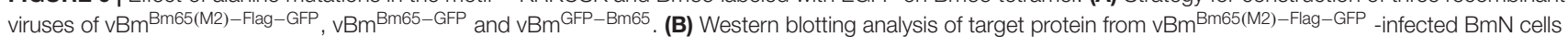
using the antibodies against the flag sequence. (C) Western blotting analysis of target protein from vBm ${ }^{\text {Bm65-GFP }}$-infected BmN cells using the antibodies against

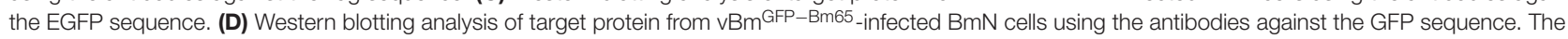
prestained protein standards are on the left. Times hpi are indicated above the lanes.

transfected with the Bm65KO and BM65(M2) constructs, the level of fluorescence was significantly lower than that of the control viruses (Figure 8B). Additionally, the transfected supernatants were harvested at $96 \mathrm{hpt}$, and used to infect $\mathrm{BmN}$ cells at a multiplicity of infection of 5 . No difference was observed between the control viruses $\mathrm{vBm}^{\mathrm{Bm} 65-\mathrm{Flag}-\mathrm{GFP}}$ and $\mathrm{vBm}^{\mathrm{WT}-\mathrm{GFP}}$, but a $90 \%$ reduction on the fluorescence level was detected for $\mathrm{vBm}^{\mathrm{Bm} 65 \mathrm{KO}-\mathrm{GFP}}$ and $\mathrm{vBm}^{\mathrm{Bm}} 65$ (M2)-Flag-GFP, compared with the controls (data not shown). To confirm the role of Bm65 during viral propagation, the supernatants containing infectious virions were harvested from $\mathrm{BmN}$ cells transfected with each of the four bacmids at $24,48,72$, and $96 \mathrm{hpt}$, and were used to infect $\mathrm{BmN}$ cells to compare the infectivity of $\mathrm{vBm}^{\mathrm{WT}-\mathrm{GFP}}, \mathrm{Bm}^{\mathrm{Bm}} 65 \mathrm{KO}-\mathrm{GFP}, \mathrm{vBm}^{\mathrm{Bm}} 65-\mathrm{Flag}-\mathrm{GFP}$, and $\mathrm{vBm}^{\mathrm{Bm} 65(\mathrm{M} 2)-\mathrm{Flag}-\mathrm{GFP}}$ by analysis of the growth curves. The assay of $\mathrm{TCID}_{50}$ endpoint dilution on $\mathrm{BmN}$ cells was performed to determine BV titer. The results showed a steady increase in virus production and the same kinetics of growth for $\mathrm{vBm}^{\mathrm{WT}-\mathrm{GFP}}$ and $\mathrm{vBm}^{\mathrm{Bm} 65-\mathrm{Flag}-\mathrm{GFP}}$. In contrast, recombinant viruses of $\mathrm{vBm}^{\mathrm{Bm} 65 \mathrm{KO}-\mathrm{GFP}}$ and $\mathrm{vBm}^{\mathrm{Bm} 65(\mathrm{M} 2)-\mathrm{Flag}-\mathrm{GFP}}$ showed reduced viral growth. According to the viral growth curve, the four viruses all showed an increased tendency in the slope of the growth curves, but the proliferation rate of $\mathrm{vBm}^{\mathrm{Bm} 65 \mathrm{KO}-\mathrm{GFP}}$ and $\mathrm{vBm}^{\mathrm{Bm}} 65(\mathrm{M} 2)-\mathrm{Flag}-\mathrm{GFP}$ are obviously slower than that of the $\mathrm{vBm} \mathrm{m}^{\mathrm{WT}-\mathrm{GFP}}$ or $\mathrm{vBm}^{\mathrm{Bm} 65-\mathrm{Flag}-\mathrm{GFP}}$ (Figure 8C). Statistical analysis revealed that there was a significant difference of the viral titers between $\mathrm{Bm} 65 \mathrm{KO}$ or BM65(M2) and Bm65 wild type virus from 48 to $96 \mathrm{hpi}$. Additionally, the viruses expressing GFP-tagged Bm65 and GFP-tagged Bm65 mutant $1\left({ }^{33}\right.$ RRIK/AAAA) were constructed in our lab and they grew about as well as the wild type (Data not shown). The results confirmed that Bm65 is not essential for viral replication, but deletion of Bm65 and alanine mutations in the motif ${ }^{76}$ KRKCSK significantly decreased the levels of infectious virions. As is well-known, the generation of progeny virions are involved with some steps, including virion assembly within nucleus, nuclear egress of assembled virions and budding from host cells. However, it is still unclear in which step was affected by the Bm65 mutant for the impairment of progeny production. 

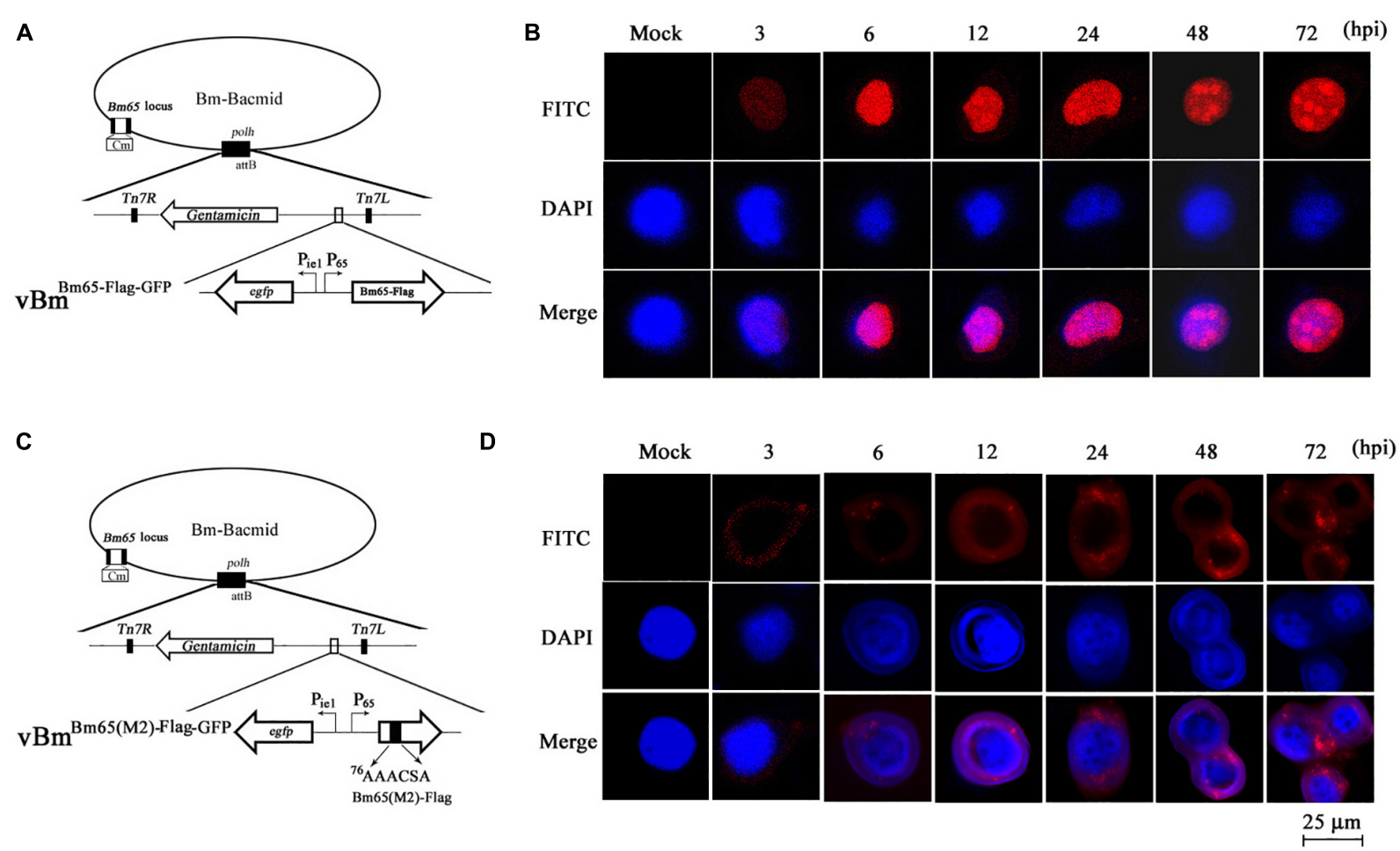

FIGURE 7 | Subcellular localization of Bm65-flag and Bm65(M2)-flag in virus-infected BmN cells. (A) Schematic diagram for preparation of recombinant virus

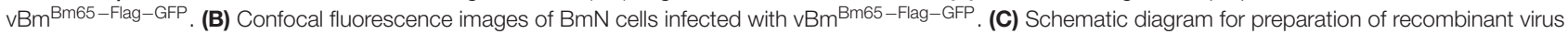

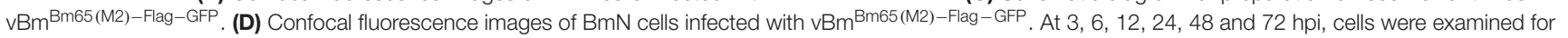
red fluorescence by confocal microscopy. From the top to the bottom: red fluorescence, DAPI-treated nucleus and the merged images.

\section{DISCUSSION}

Nuclear proteins are translated from mRNA in cytoplasm and must be transported to nucleus where they exert their biological functions. It is generally accepted that a protein is targeted to nucleus by classical NLSs, which can be divided into two categories of monopartite and bipartite sequences. For example, the PKKKRKV sequence of simian virus 40 (SV40) large T antigen is a monopartite sequence (Kalderon et al., 1984), and the KRPAATKKAGQAKKKK sequence of nucleophosmin is a bipartite sequence (Robbins et al., 1991). The classical NLSs are directed to form a heterotrimeric complex including the cargo protein and two importins, which further mediates transport of the heterotrimer into nucleus by its affinity with nucleoporins (Ben-Efraim and Gerace, 2001). Some baculovirus proteins have also been reported to contain the classical NLSs. For example, the monopartite motif of classical NLSs was discovered in baculovirus polyhedrin and AcMNPV BV/ODV-c42 (Jarvis et al., 1991; Wang et al., 2008), and residues 804-827 of AcMNPV DNApol are a typical bipartite NLS motif (Feng and Krell, 2014). Additionally, some non-canonical NLSs (nNLSs) have also been identified in baculovirus proteins, which do not correspond with the prototypical NLS consensus sequence. For example, Guo et al. (2010) reported that two basic residue clusters at positions 117-120 ( ${ }^{117}$ RKRR) and $144-148$ ( ${ }^{144}$ RKR-K) constituted an authentic NLS for mediating nuclear localization of Bm47.
A novel localization signal containing $K(75) / R(76), H(81)$, $K(83) / R(84), R(87)$, and $K(100)$ was scattered in the different positions of BmNPV LEF-11, which was identified to be critical for the nuclear localization of target protein (Zhang et al., 2014). The residues KKIREN of LEF-3 have been identified as a core NLS required for nuclear transport, which was further improved by other adjacent positively charged residues (Au et al., 2009).

Previous studies revealed that Bm65 accumulated mainly within the nucleus, and was involved with the repair of UVCinduced DNA damage (Tang et al., 2015, 2017). Moreover, Ac79, a homolog of Bm65, is responsible to encode an early gene product that are structurally similar to UvrC and intronencoded endonucleases, which was required for efficient BV production (Wu and Passarelli, 2012). Alignment result showed $99 \%$ sequence identity with the amino acid of Bm65. The high similarity of sequence implied that the role of Bm65 in viral propagation may be similar to Ac79. However, the mechanism by which Bm65 is transported into the nucleus of virus-infected cells and the failure of nuclear import of Bm65 on viral production are still unclear. In the study, flag-labeled Bm65 is under the control of Bm65 native promoter in the study (Figures 2, 8), and it was detected from 3 to $72 \mathrm{hpi}$ (Figures 2B-D). So, we think that Bm65 native promoter is similar to iel promoter, and it can function like the iel promoter. To accelerate the progress on Bm65 research, some plasmids 


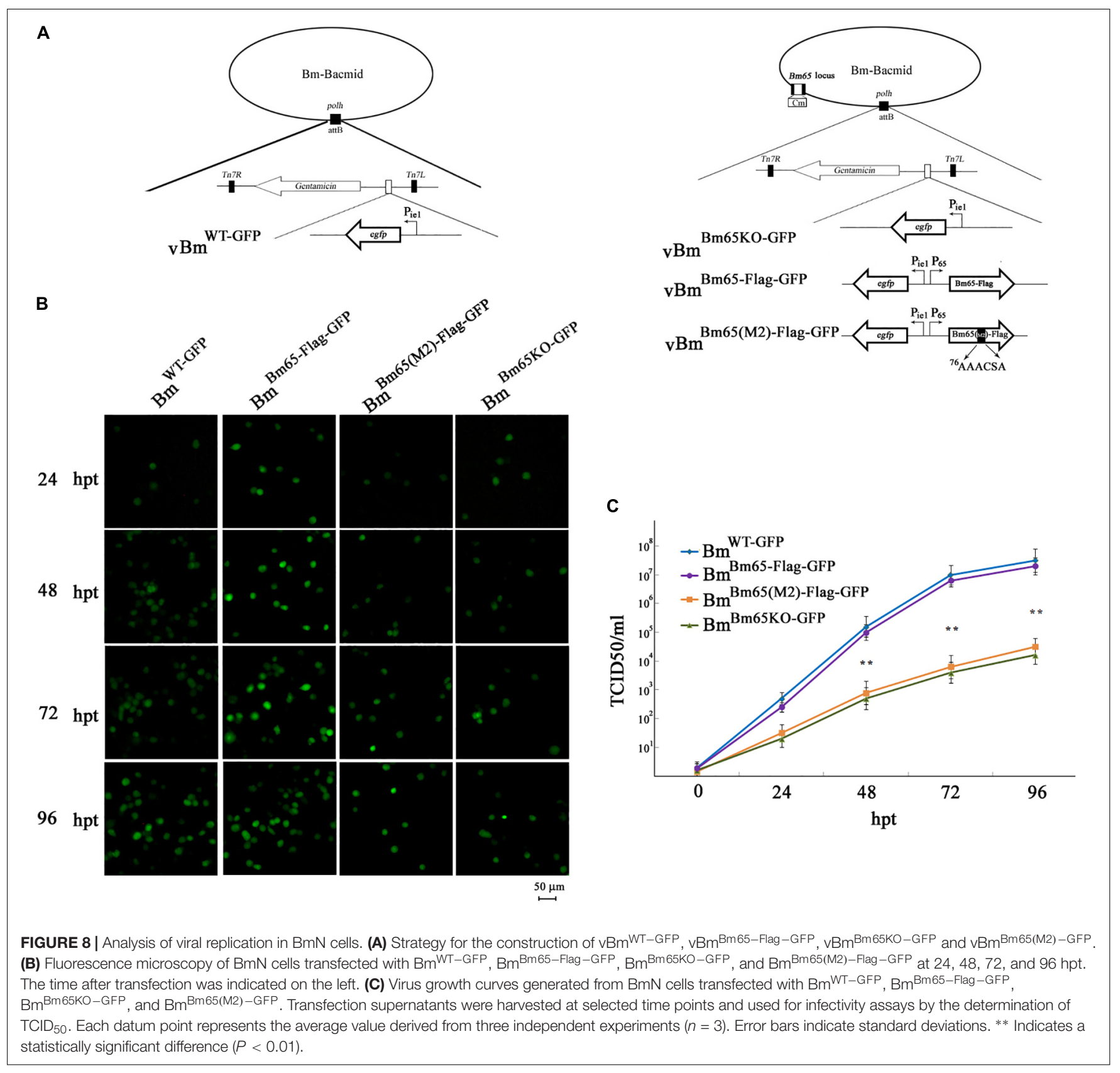

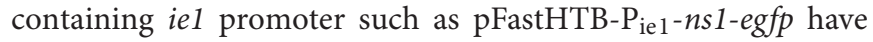
been constructed and conserved in our laboratory, which can be used for the transient expression analysis of $\mathrm{Bm} 65$ in $\mathrm{BmN}$ cells. So, ie1 promoter was chosen to drive the expression based on the convenience and economic benefits in the study. A series of truncations and arginine/lysine-to-alanine mutations severely impaired the nuclear import of Bm65. The ${ }^{76} \mathrm{KRKCSK}$ motif of Bm65 was identified as a novel NLS required for nuclear import of Bm65, but another basic cluster of ${ }^{33}$ RRIK was identified as unnecessary for the nuclear import of Bm65. Furthermore, the leucine-rich cluster of ${ }^{92}$ PLLLHKFLL may function as a nuclear export signal for maintaining the dynamic balance of Bm65 between nucleus and cytoplasm.
Previous research has revealed that $B m 65$ is not essential for viral propagation (Ono et al., 2012). However, Tang et al. (2013) reported that Bm65 was an essential gene for viral propagation. In the present study, Bm65 was identified as an unnecessary gene for viral propagation. In Tang et al. (2013), Bm65 was regarded to be an essential gene for viral propagation, which may be resulted from the failure of transfection of $\mathrm{BmN}$ cells. As we know, donor plasmid of pFB-ieGP-Bm65 was transformed into electrocompetent $\mathrm{DH} 10 \mathrm{~B}$ cells containing $\mathrm{Bm}^{\mathrm{Bm} 65 \mathrm{KO}}$, which was used for the generation of $\mathrm{Bm}^{\mathrm{Bm} 65 \mathrm{KO}-\mathrm{GP}}$ by transposition. Furthermore, the recombinant $\mathrm{Bm}^{\mathrm{Bm} 65 \mathrm{KO}-\mathrm{GP}}$ extracted from $\mathrm{DH} 10 \mathrm{~B}$ cells was used for transfection of $\mathrm{BmN}$ cells. However, the pFB-ieGP-Bm65 was also extracted from 
DH10B cells besides the $\mathrm{Bm}^{\mathrm{Bm} 65 \mathrm{KO}-\mathrm{GP}}$, and the abundance of pFB-ieGP-Bm65 is more than that of $\mathrm{Bm}^{\mathrm{Bm} 65 \mathrm{KO}-\mathrm{GP}}$ in the extracted DNA. Fluorescence signal can be observed in $\mathrm{BmN}$ cells transfected with pFB-ieGP-Bm65, but no infectious virions were produced in the transfection supernatant. When the transfection supernatant was collected and further used for infection of $\mathrm{BmN}$ cells, it can not initiate the second infection of $\mathrm{BmN}$ cells and no fluorescence signal was observed in $\mathrm{BmN}$ cells. So, Tang et al. (2013) regarded that Bm65 was an essential gene for viral propagation by mistake. In fact, infectious virions can be produced in $\mathrm{BmN}$ cells transfected with $\mathrm{Bm}^{\mathrm{Bm} 65 \mathrm{KO}-\mathrm{GP}}$. Furthermore, the recombinant virus with deletion of Bm65 was confirmed to be infectious by passage analysis in $\mathrm{BmN}$ cells, but the analysis of viral titer showed that the deletion significantly decreased the production of infectious virions (Figure 8C). So, Bm65 was not an essential gene for viral propagation in the study, but it can regulate the production of viral propagation.

Furthermore, mutations in the motif of ${ }^{76} \mathrm{KRKCSK}$ resulted in the cytoplasmic accumulation of Bm65. Compared with wildtype Bm65, the mutations greatly decreased the production of infectious virions. Bm65 was first identified to form a stable sodium dodecyl sulfate (SDS)-resistant tetramer in the natural state, and there was some fluorescent aggregates in cytoplasm and nucleus when alanine mutations were made in the motif of ${ }^{76} \mathrm{KRKCSK}$. Compared with wild type of Bm65 (Figure 5D), fluorescent aggregates caused by the mutant type of Bm65 are accumulated exclusively in the cytoplasm of virusinfected BmN cells (Figure 5B), and the fluorescent aggregates observed in wild type and mutant Bm65 virus-infected cells vary in the sizes and levels. It is supposed that Bm65 tetramer may increase the possibility of the formation of fluorescent aggregates. Of course, the misfolding of Bm65 is an important factor for the formation of protein aggregates. To the best of our knowledge, some baculovirus proteins such as LEF-10 and LEF-11 form protein aggregates (Dong et al., 2015; Xu et al., 2016). Moreover, amyloid $\beta$-protein (Walsh et al., 1999; Rangachari et al., 2007), sup35 (Kryndushkin et al., 2003), and $\alpha$-synuclein (Rekas et al., 2010) are able to form SDS-resistant aggregates. Protein aggregation is a natural phenomenon that occurs both in vitro and in vivo. However, it is generally regarded to be harmful and has been implicated in a wide variety of diseases such as neurodegenerative diseases, and the possible correlations between protein aggregates and diseases deserves our deep consideration. Besides the tetrameric form from 3 to $96 \mathrm{hpi}$, a major lower molecular weight band at about $26 \mathrm{kDa}$ was also examined in Figure 2D from 48 to 72 hpi, indicating that it may be a Bm65 dimer. The mutations of cysteine residues at positions 12, 46, and 79 did not affect the formation of Bm65 tetramer, but they directly resulted in increased instability of Bm65 tetramer in late infection. Additionally, some unexpected bands around $26 \mathrm{kDa}$ and below $26 \mathrm{kDa}$ were examined in Figures 6C,D. The abundance of the lower molecular weight proteins is so rich, but the synthesis mechanism is still unclear. Therefore, the possibility of Bm65 tetramer by disulfide linkage formation was precluded by the mutational analysis.
Additionally, recombinant plasmid of pET-30a-Bm65 was constructed by Tang et al. (2015) and transformed into BL21 cells for expression of $6 \times$ His-Bm65. Western blotting result indicated that only $6 \times$ His-Bm65 monomer was present in the soluble supernatant from the lysates of BL21 cells. Moreover, a lot of Bm65 inclusion body was found to be produced in E. coli. Therefore, we think that it is unfavorable for the formation of Bm65 tetramer in E. coli strains. Compared with the monomer in E. coli, Bm65 tetramer was examined in $\mathrm{vBm}^{\mathrm{Bm} 65-\mathrm{Flag}-\mathrm{GFP}}$-infected BmN cells, but GFP-tagged Bm65 monomer was examined in virus-infected $\mathrm{BmN}$ cells in the study (Figures 6C,D). It was regarded that flag tagged Bm65 is equivalent to the Bm65 native state, but the GFP tag affected the multimerization of Bm65. Moreover, the intermediate band about $26 \mathrm{kDa}$ in Figure 2D from 48 to 72 hpi may be Bm65 dimer or Bm65 protein complex. The replacement of cysteine with alanine may decreased the stability of Bm65 tetramer, which was involved with the generation of the intermediate. Additionally, the formation of Bm65 tetramer may need the assistance from other proteins in BmNPVinfected BmN cells. Tang et al. (2015) reported the monomersonly, which was obtained in the soluble supernatant from the lysates of BL21 cells. In the study, Bm65 tetramer was easily produced in BmNPV-infected $\mathrm{BmN}$ cells, which has nothing to do with disulfide linkage. Some mutations in the motif of ${ }^{76} \mathrm{KRKCSK}$ resulted in failure of the nuclear import of Bm65, which was involved with the decreased production of infectious virions. Moreover, we found that there are a lot of hydrophobic amino acids scattered in the sequence of Bm65, which may be the direct reason for the formation of Bm65 tetramer. Further research is required to disclose the formative mechanism of Bm65 tetramer. Meanwhile, it is also an interesting scientific issue for further investigation whether these hydrophobic amino acids are positively selected during virus/host coevolution.

\section{DATA AVAILABILITY STATEMENT}

The datasets generated for this study are available on request to the corresponding author.

\section{AUTHOR CONTRIBUTIONS}

GL designed the study, researched, and analyzed the data. XQ, $\mathrm{HC}$, and $\mathrm{LD}$ researched the data. FC, ZG, and $\mathrm{ZH}$ contributed to the discussion. KC provided financial assistance. QT designed the study and wrote the manuscript.

\section{FUNDING}

This study was supported by the National Natural Science Foundation of China (Nos. 31402016, 31770174, 31570150, 81402840, and 31861143051). 


\section{REFERENCES}

Au, V., Yu, M., and Carstens, E. B. (2009). Characterization of a baculovirus nuclear localization signal domain in the late expression factor 3 protein. Virology 385, 209-217. doi: 10.1016/j.virol.2008.10.051

Ben-Efraim, I., and Gerace, L. (2001). Gradient of increasing affinity of importin beta for nucleoporins along the pathway of nuclear import. J. Cell Biol. 152, 411-417. doi: 10.1083/jcb.152.2.411

Cheng, X. W., and Lynn, D. E. (2009). Baculovirus interactions in vitro and in vivo. Adv. Appl. Microbiol. 68, 217-239. doi: 10.1016/S0065-2164(09)01205-1202

Clem, R. J., and Passarelli, A. L. (2013). Baculoviruses: sophisticated pathogens of insects. PLoS Pathog. 9:e1003729. doi: 10.1371/journal.ppat.1003729

Dong, Z. Q., Hu, N., Zhang, J., Chen, T. T., Cao, M. Y., Li, H. Q., et al. (2015). Oligomerization of baculovirus LEF-11 is involved in viral DNA replication. PLoS One 10:e0144930. doi: 10.1371/journal.pone.0144930

Feng, G., and Krell, P. J. (2014). Autographa californica multiple nucleopolyhedrovirus DNA polymerase $\mathrm{C}$ terminus is required for nuclear localization and viral DNA replication. J. Virol. 88, 10918-10933. doi: 10.1128/JVI.01167-1114

Gomi, S., Majima, K., and Maeda, S. (1999). Sequence analysis of the genome of Bombyx mori nucleopolyhedrovirus. J. Gen. Virol. 80, 1323-1337. doi: 10.1099/ 0022-1317-80-5-1323

Guo, Z. J., Wang, D. X., Yao, Q., Chen, K. P., and Zhang, C. X. (2010). Identification of a novel functional nuclear localization signal in the protein encoded by open reading frame 47 of Bombyx mori nucleopolyhedrovirus. Arch. Virol. 155, 1943-1950. doi: 10.1007/s00705-010-0782-1

Herniou, E. A., Olszewski, J. A., Cory, J. S., and O’Reilly, D. R. (2003). The genome sequence and evolution of baculoviruses. Annu. Rev. Entomol. 48, 211-234. doi: 10.1146/annurev.ento.48.091801.112756

Jarvis, D. L., Bohlmeyer, D. A., and Garcia, A. Jr. (1991). Requirements for nuclear localization and supramolecular assembly of a baculovirus polyhedrin protein. Virology 185, 795-810. doi: 10.1016/0042-6822(91)90551-L

Kalderon, D., Roberts, B. L., Richardson, W. D., and Smith, A. E. (1984). A short amino acid sequence able to specify nuclear location. Cell 39, 499-509. doi: 10.1016/0092-8674(84)90457-4

Kryndushkin, D. S., Alexandrov, I. M., Ter-Avanesyan, M. D., and Kushnirov, V. V. (2003). Yeast [PSI+] prion aggregates are formed by small Sup35 polymers fragmented by Hsp104. J. Biol. Chem. 278, 49636-49643. doi: 10.1074/jbc. M307996200

Li, G., Li, M., Xu, W., Zhou, Q., Hu, Z., Tang, Q., et al. (2015). Regulation of BmBDV NS1 by phosphorylation: impact of mutagenesis at consensus phosphorylation sites on ATPase activity and cytopathic effects. J. Invertebr. Pathol. 133, 66-72. doi: 10.1016/j.jip.2015.12.006

Li, G., Wang, P., Li, M., Xu, W., Hu, Z., and Yao, Q. (2014). Expression of ns1 gene from Bombyx mori bidensovirus by a novel baculovirus expression system. Sheng Wu Gong Cheng Xue Bao 30, 625-635.

Ono, C., Kamagata, T., Taka, H., Sahara, K., Asano, S., and Bando, H. (2012). Phenotypic grouping of $141 \mathrm{BmNPVs}$ lacking viral gene sequences. Virus Res. 165, 197-206. doi: 10.1016/j.virusres.2012.02.016

O'Reilly, D. R., Miller, L. K., and Luckow, V. A. (1994). Baculovirus Expression Vectors: A Laboratory Manual. New York, NY: Oxford University Press.

Qin, L., Xia, H., Shi, H., Zhou, Y., Chen, L., Yao, Q., et al. (2012). Comparative proteomic analysis reveals that caspase- 1 and serine protease may be involved in silkworm resistance to Bombyx mori nuclear polyhedrosis virus. J. Proteomics. 75, 3630-3638. doi: 10.1016/j.jprot.2012.04.015

Rangachari, V., Moore, B. D., Reed, D. K., Sonoda, L. K., Bridges, A. W., Conboy, E., et al. (2007). Amyloid-beta(1-42) rapidly forms protofibrils and oligomers by distinct pathways in low concentrations of sodium dodecylsulfate. Biochemistry 46, 12451-12462. doi: 10.1021/bi701213s

Rekas, A., Knott, R. B., Sokolova, A., Barnham, K. J., Perez, K. A., Masters, C. L., et al. (2010). The structure of dopamine induced alpha-synuclein oligomers. Eur. Biophys. J. 39, 1407-1419. doi: 10.1007/s00249-010-0595-x

Robbins, J., Dilworth, S. M., Laskey, R. A., and Dingwall, C. (1991). Two interdependent basic domains in nucleoplasmin nuclear targeting sequence: identification of a class of bipartite nuclear targeting sequence. Cell 64, 615-623. doi: 10.1016/0092-8674(91)90245-T

Rohrmann, G. F. (2013). Baculovirus Molecular Biology, 3rd Edn. Bethesda, MD: National Library of Medicine, National Center for Biotechnology Information (US).

Slack, J., and Arif, B. M. (2007). The baculoviruses occlusion-derived virus: virion structure and function. Adv. Virus Res. 69, 99-165. doi: 10.1016/S0065-3527(06) 69003-9

Tang, Q., Hu, Z., Yang, Y., Wu, H., Qiu, L., Chen, K., et al. (2015). Overexpression of Bm65 correlates with reduced susceptibility to inactivation by UV light. J. Invertebr. Pathol. 127, 87-92. doi: 10.1016/j.jip.2015. 03.003

Tang, Q., Li, G., Yao, Q., Chen, L., Feng, F., Yuan, Y., et al. (2013). Bm65 is essential for the propagation of Bombyx mori nucleopolyhedrovirus. Curr. Microbiol. 66, 22-29. doi: 10.1007/s00284-012-0236-y

Tang, Q., Wu, P., Hu, Z., Yang, Y., Qiu, L., Liu, H., et al. (2017). Evidence for the role of BmNPV Bm65 protein in the repair of ultraviolet-induced DNA damage. J. Invertebr. Pathol. 149, 82-86. doi: 10.1016/j.jip.2017.08.004

van Oers, M. M., and Vlak, J. M. (2007). Baculovirus genomics. Curr. Drug Targets 8, 1051-1068. doi: 10.2174/138945007782151333

Walsh, D. M., Hartley, D. M., Kusumoto, Y., Fezoui, Y., Condron, M. M., Lomakin, A., et al. (1999). Amyloid beta-protein fibrillogenesis. Structure and biological activity of protofibrillar intermediates. J. Biol. Chem. 274, 25945-25952. doi: 10.1074/jbc.274.36.25945

Wang, Y., Wang, Q., Liang, C., Song, J., Li, N., Shi, H., et al. (2008). Autographa californica multiple nucleopolyhedrovirus nucleocapsid protein BV/ODV-C42 mediates the nuclear entry of P78/83. J. Virol. 82, 4554-4561. doi: 10.1128/JVI. 02510-07

Wu, W., and Passarelli, A. L. (2012). The Autographa californica M nucleopolyhedrovirus ac79 gene encodes an early gene product with structural similarities to UvrC and intron-encoded endonucleases that is required for efficient budded virus production. J. Virol. 86, 5614-5625. doi: 10.1128/JVI.06252-11

Xu, X., Zhou, X., Nan, H., Zhao, Y., Bai, Y., Ou, Y., et al. (2016). Aggregation of AcMNPV LEF-10 and its impact on viral late gene expression. PLoS One 11:e0154835. doi: 10.1371/journal.pone.0154835

Xue, J., Qiao, N., Zhang, W., Cheng, R. L., Zhang, X. Q., Bao, Y. Y., et al. (2012). Dynamic interactions between Bombyx mori nucleopolyhedrovirus and its host cells revealed by transcriptome analysis. J. Virol. 86, 7345-7359. doi: 10.1128/ JVI.07217-12

Yao, Z., Guo, Z., Wang, Y., Li, W., Fu, Y., Lin, Y., et al. (2019). Integrated succinylome and metabolome profiling reveals crucial role of S-Ribosylhomocysteine lyase in quorum sensing and metabolism of aeromonas hydrophila. Mol. Cell Proteomics 18, 200-215. doi: 10.1074/mcp.RA118.00 1035

Yin, F., Zhu, Z., Liu, X., Hou, D., Wang, J., Zhang, L., et al. (2015). The complete genome of a new betabaculovirus from Clostera anastomosis. PLoS One 10:e0132792. doi: 10.1371/journal.pone.0132792

Zhang, J., Dong, Z. Q., Zhang, C. D., He, Q., Chen, X. M., Cao, M. Y., et al. (2014). Identification of a novel nuclear localization signal of baculovirus late expression factor 11. Virus Res. 184, 111-119. doi: 10.1016/j.virusres.2014.02. 020

Conflict of Interest: The authors declare that the research was conducted in the absence of any commercial or financial relationships that could be construed as a potential conflict of interest.

Copyright (c) 2019 Li, Qi, Chen, Hu, Chen, Deng, Guo, Chen and Tang. This is an open-access article distributed under the terms of the Creative Commons Attribution License (CC BY). The use, distribution or reproduction in other forums is permitted, provided the original author(s) and the copyright owner(s) are credited and that the original publication in this journal is cited, in accordance with accepted academic practice. No use, distribution or reproduction is permitted which does not comply with these terms. 